\title{
Thioesterase-mediated control of cellular calcium homeostasis enables hepatic ER stress
}

\author{
Baran A. Ersoy, Kristal M. Maner-Smith, Yingxia Li, Ipek Alpertunga, and David E. Cohen \\ Department of Medicine, Division of Gastroenterology and Hepatology, Weill Cornell Medical College, New York, New York, USA.
}

\begin{abstract}
The incorporation of excess saturated free fatty acids (SFAs) into membrane phospholipids within the ER promotes ER stress, insulin resistance, and hepatic gluconeogenesis. Thioesterase superfamily member 2 (Them2) is a mitochondriaassociated long-chain fatty acyl-CoA thioesterase that is activated upon binding phosphatidylcholine transfer protein (PC-TP). Under fasting conditions, the Them2/PC-TP complex directs saturated fatty acyl-CoA toward $\beta$-oxidation. Here, we showed that during either chronic overnutrition or acute induction of ER stress, Them2 and PC-TP play critical roles in trafficking SFAs into the glycerolipid biosynthetic pathway to form saturated phospholipids, which ultimately reduce ER membrane fluidity. The Them2/PC-TP complex activated ER stress pathways by enhancing translocon-mediated efflux of ER calcium. The increased cytosolic calcium, in turn, led to the phosphorylation of calcium/calmodulin-dependent protein kinase II, which promoted both hepatic insulin resistance and gluconeogenesis. These findings delineate a mechanistic link between obesity and insulin resistance and establish the Them2/PC-TP complex as an attractive target for the management of hepatic steatosis and insulin resistance.
\end{abstract}

\section{Introduction}

Hepatic lipotoxicity due to saturated free fatty acids (SFAs) is a key contributor to the insulin resistance and excess hepatic glucose production associated with type 2 diabetes mellitus (1-3). SFAs are rapidly incorporated into ER membrane phospholipids $(4,5)$, the fatty acid composition of which is dynamic and dependent on nutritional status. Phospholipids containing saturated fatty acyl chains promote ER stress (5), at least in part by increasing calcium efflux from the ER lumen (4). If unresolved, ongoing ER stress promotes insulin resistance and excess hepatic gluconeogenesis (6).

Plasma nonesterified fatty acids (NEFAs) originating from the diet or adipose tissue stores are taken up by the liver or are synthesized de novo by well-described, transcriptionally regulated pathways $(7,8)$. Within the liver, NEFAs are esterified to form fatty acyl-CoA molecules, the metabolic fates of which depend on their intracellular trafficking controlled by compartmentalized hydrolysis and re-esterification by long-chain acyl-CoA thioesterases (Acots) and synthetases (ACSLs), respectively (9-11). For example, mitochondria-associated ACSL1 directs fatty acyl-CoAs toward either $\beta$-oxidation or glycerolipid biosynthesis, depending on metabolic conditions within the hepatocyte $(11,12)$.

We have identified thioesterase superfamily member 2 (Them2; synonym Acot13) as a protein that interacts with phosphatidylcholine transfer protein (PC-TP; synonym StARD2) (13). Them 2 associates with mitochondria and exhibits substrate specificity for long-chain saturated fatty acyl-CoA (e.g., myristoylCoA and palmitoyl-CoA) $(14,15)$. PC-TP comprises a single lipidbinding domain with high specificity for phosphatidylcholines

Conflict of interest: The authors have declared that no conflict of interest exists.

Submitted: February 10, 2017; Accepted: October 12, 2017.

Reference information: J Clin Invest. 2018;128(1):141-156.

https://doi.org/10.1172/JCI93123.
(PCs), particularly those containing more unsaturated fatty acyl chains (13). Binding of PCs to PC-TP promotes its interaction with Them2 (16), which in turn increases thioesterase activity (13). Mice lacking either Them2 (Them2-/-) or PC-TP $\left(\right.$ Pctp $\left.^{-/}\right)$exhibit altered fatty acid metabolism $(15,17,18)$. In keeping with a primary role in SFA trafficking, the Them $2 / \mathrm{PC}-\mathrm{TP}$ complex promotes $\beta$-oxidation of exogenous NEFAs in cultured mouse hepatocytes under fasting conditions (19).

The genetic ablation of Them 2 or PC-TP also increases hepatic insulin sensitivity and protects against high-fat diet-induced increases in hepatic glucose production by incompletely understood mechanisms $(15,17)$. We have postulated that this complex channels fatty acids to glycerol-phosphate acyl transferase 1 (GPAT1), a mitochondria-associated enzyme that represents the rate-controlling step in glycerolipid synthesis, including ER membrane phospholipids (20). Our current findings demonstrate that, by promoting the incorporation of SFAs into ER membrane phospholipids, the Them2/PC-TP complex reduces membrane fluidity, increases calcium efflux through translocons within the ER membrane, and activates ER stress pathways that promote insulin resistance and excess hepatic glucose production.

\section{Results}

Critical contributions of Them2 and PC-TP to ER stress in liver. Because Them $2^{-/-}$and $P c t p^{-/-}$mice are each protected against highfat diet-induced insulin resistance, which can be a manifestation of chronic ER stress (21), we investigated the respective contributions of Them 2 and PC-TP to ER stress in response to high-fat feeding (Figure 1A and Supplemental Figure 1, A-C; supplemental material available online with this article; https://doi.org/10.1172/ JCI93123DS1). The absence of Them 2 or PC-TP expression was associated with reduced hepatic expression of activated (i.e., phosphorylated) PKR-like ER kinase (p-PERK), glucose-regulated 


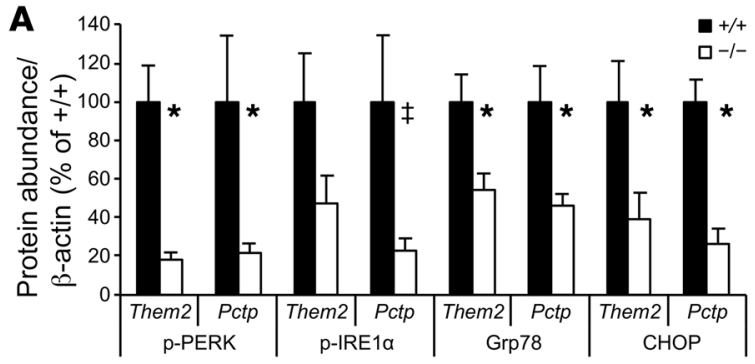

D

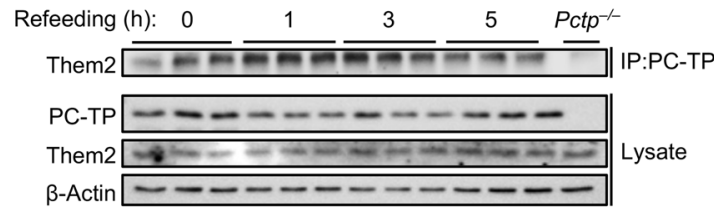

B
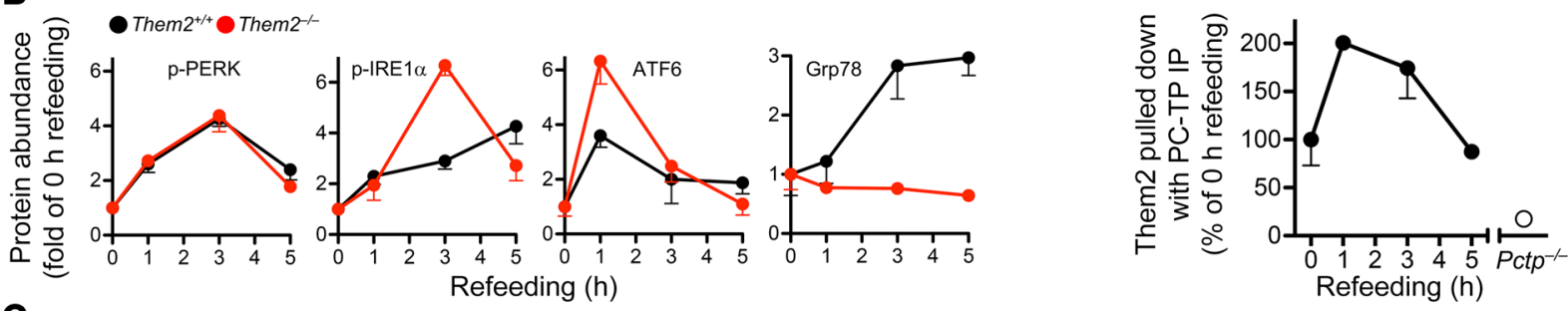

C
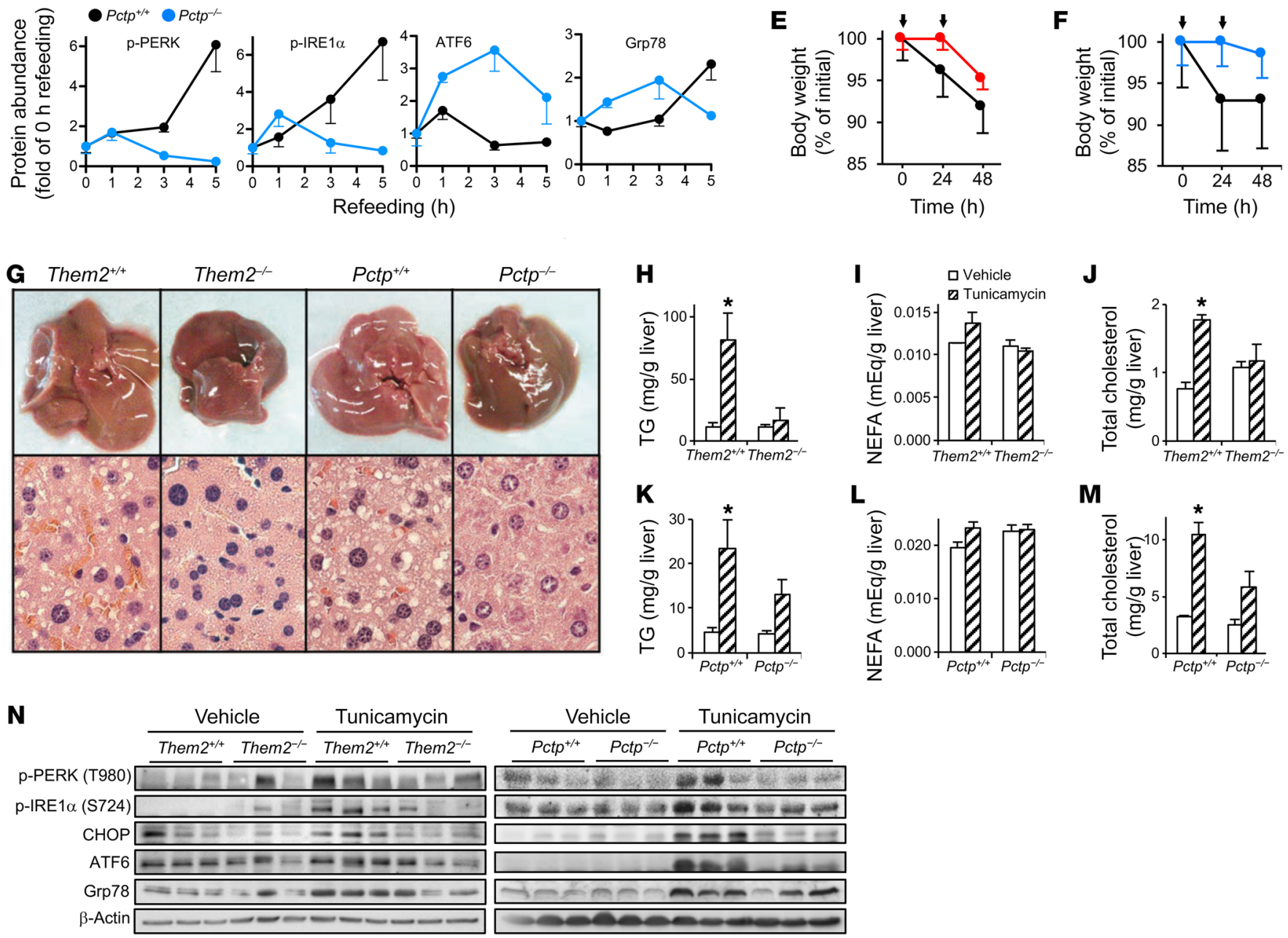
Figure 1. Them2 and PC-TP regulate ER stress in mice. (A) The livers of Them2 $2^{-/-}$and $P \mathrm{Ctp}^{-/-}$mice are protected against ER stress due to high-fat feeding. Liver homogenates from high-fat-fed Them $2^{+/+}(n=8)$, Them2 $2^{-/-}(n=9), \operatorname{Pctp}^{+/+}(n=5)$, and Pctp ${ }^{-/-}(n=5)$ mice were subjected to immunoblot analyses, and bands were quantified by densitometry and normalized to $\beta$-actin. Error bars represent SEM. ${ }^{*} P<0.05$ compared with Them2 $2^{-/-}$or $P c t p^{+/+},{ }^{\ddagger} P=0.057$ compared with $P c t p^{+/+}$. (B and C) Them2 and PC-TP modulate the resolution of hepatic ER stress following refeeding. Liver homogenates ( $n=3$ per genotype) were subjected to immunoblot analyses, and bands were quantified by densitometry. (D) The influence of refeeding on the interactions between PC-TP and Them 2 was determined by coimmunoprecipitation in the liver lysates that were harvested from $\operatorname{Pctp}^{+/+}(n=3)$ mice, and bands were quantified by densitometry. (E-N) Them2 or PC-TP expression enables tunicamycin-induced liver injury. Eight-week-old chow-fed mice were injected i.p. with tunicamycin $(0.25$ $\mathrm{mg} / \mathrm{kg}$ body weight) or vehicle (DMSO, $0.25 \% \mathrm{v} / \mathrm{v}$ ) for 2 consecutive days. ( $E$ and $\mathbf{F}$ ) Body weights ( $n=3$ per genotype) following tunicamycin injections (arrows). (G) Livers were harvested and imaged 6 hours following food restriction. Liver sections were subjected to hematoxylin and eosin (H\&E) staining. Microscope images ( $\times 60)$ are representatives of $n=3$. (H-M) Livers were analyzed for the concentrations of triglycerides (TC) ( $\mathbf{H}$ and $\mathbf{K}$ ), NEFA (I and $\mathbf{L}$ ), and total cholesterol (J and $\mathbf{M})$. ( $\mathbf{N}$ ) ER stress markers were detected in the liver lysates by immunoblot analyses. Error bars represent SEM. ${ }^{*} P<0.05$ compared with WT. Statistical significance was determined by Student's $t$ test.

protein 78 (Grp78), and CCAAT/enhancer-binding protein homologous protein (CHOP) and a trend toward decreased expression of phosphorylated inositol-requiring enzyme $1 \alpha$ (p-IRE1 $\alpha)$. Cleaved activating transcription factor 6 (ATF6) levels were also decreased in livers of $\mathrm{Pctp}^{-/-}$but not Them $2^{-/-}$mice.

ER stress is also induced when chow-fed mice are re-fed after a prolonged fast. Unlike in livers of high-fat-fed mice, ER stress induced by so-called fasting-refeeding is self-limited, resolves upon restoration of nutrient balance, and does not impair long-term glucose homeostasis (22). Although the time course of PERK activation and resolution was independent of Them 2 expression (Figure 1B and Supplemental Figure 1D), the magnitude of PERK activation was reduced in livers of Them $2^{-1-}$ mice (Supplemental Figure 1E). In addition to reducing p-PERK, the absence of PC-TP expression led to its more rapid return to baseline (Figure 1C and Supplemental Figure 1F). In livers of WT mice, $p$-IRE1 $\alpha$ levels were elevated throughout the 5-hour refeeding period (Figure 1, B and C, and Supplemental Figure 1, D and F). Although p-IRE1 $\alpha$ levels were more elevated in Them $2^{-/-}$livers at 3 hours (Supplemental Figure 1E), they returned to baseline by 5 hours (Figure 1B and Supplemental Figure 1D). Similarly, in Pct $p^{-/-}$livers, p-IRE1 $\alpha$ was elevated early within 1 hour of refeeding and returned to baseline by 3 hours (Figure 1C and Supplemental Figure 1F). Livers of Them $2^{-/}$and $\mathrm{Pctp}^{-/-}$mice exhibited robust ATF6 cleavage within 1 hour of refeeding, whereas weaker ATF6 activation in livers of WT mice subsided within 3 hours (Figure 1, B and C, and Supplemental Figure 1, D and F). Grp78 expression also exhibited time-dependent elevations in livers of WT mice, which did not resolve in 5 hours (Figure 1, B and C, and Supplemental Figure 1, D and F). Whereas hepatic Grp78 expression remained unchanged in Them $2^{-/-}$mice (Figure 1B and Supplemental Figure 1D), initial increases in Pctp ${ }^{-/}$ mice (within 1-3 hours) were resolved within 5 hours (Figure 1C and Supplemental Figure 1F). Because Them $2^{-/-}$and Pct $^{-/-}$mice both exhibited similarly rapid resolution of hepatic ER stress, we next tested whether refeeding might influence the interaction between Them 2 and PC-TP. Indeed, more Them 2 protein was coimmunoprecipitated with PC-TP within 1-3 hours of refeeding (Figure 1D). Them2-PC-TP interactions returned their fasting levels within 5 hours (Figure 1D).

To ensure that reductions in hepatic ER stress in Them $2^{-/-}$and Pctp $^{-/}$mice were not simply attributable to improved glucose homeostasis $(15,17,18)$, ER stress was also induced chemically by i.p. administration of tunicamycin, which inhibits protein glycosylation and promotes protein misfolding (21). Treatment of mice with tunicamycin led to body weight losses of $8.1 \%$ and $7 \%$ in Them $^{+/+}$and Pctp $^{+/+}$mice, respectively (Figure 1, E and F). By contrast, Them2 $2^{-/-}$and $P c t p^{-/}$mice sustained body weight losses of only $4.3 \%$ and $1.5 \%$, respectively (Figure 1, E and F). Tunicamycin treatment tended to reduce glucose tolerance in WT but not in Them $2^{-/-}$and $\mathrm{Pctp}^{-/-}$mice (Supplemental Figure 2A). Insulin sensitivity also tended to decrease following tunicamycin injections in WT and $\mathrm{Pctp}^{-/-}$mice but not in Them $2^{-/-}$mice (Supplemental Figure 2B). The failure to reach statistical significance may have been due, at least in part, to substantial variations in body weight loss following tunicamycin treatment.

Whereas livers of $T h e m 2^{+/+}$and $P c t p^{+/+}$mice appeared grossly pale, livers from tunicamycin-treated $\mathrm{Them}^{-/-}$and $\mathrm{Pctp}^{-/-}$mice were indistinguishable from vehicle-treated WT controls (Figure $1 G$ and Supplemental Figure 3). Histologically, large lipid droplets were evident in livers of WT, but not Them $2^{-/-}$and $P c t p^{-/-}$mice (Figure $1 G$ ). Tunicamycin treatment increased hepatic triglyceride concentrations, tended to increase NEFA concentrations, and increased cholesterol concentrations in the livers of $T h e m 2^{+/+}$, but not Them $2^{-/-}$mice (Figure 1, H-J). Tunicamycin induced similar changes in the livers of Pct $\mathrm{p}^{+/+}$mice, but in the absence of PC-TP expression, we observed only slight increases in these lipid concentrations (Figure 1, K-M). Although NEFA concentrations tended to be higher in the livers of vehicle-treated $P c t p^{-/-}$mice, tunicamycin treatment did not increase concentrations relative to vehicle treatment (Figure 1L). In keeping with the likelihood that lipid accumulation in WT mice was a consequence of ER stress, we observed increased levels of p-PERK, p-IRE1 $\alpha$, CHOP, cleaved ATF6, and Grp78 in the livers of tunicamycin-treated WT mice (Figure 1N). By contrast, the levels of these ER stress markers in the livers of tunicamycin-treated Them $2^{---}$and $\mathrm{Pctp}^{-/-}$mice remained indistinguishable from those of vehicle-treated mice (Figure $1 \mathrm{~N}$ ).

To validate the importance of Them2-PC-TP interactions in regulating the ER stress response, we treated mice with the PC-TP inhibitor compound $\mathrm{A} 1(16,17,19,23)$. In Them $2^{+/+}$mice, inhibition of PC-TP by compound A1 before tunicamycin administration led to increased PERK activity and reduced activities of IRE1 $\alpha$ and eIF $2 \alpha$ relative to vehicle-treated controls (Supplemental Figure 4). Moreover, compound A1 treatment was associated with a $62 \%$ reduction in $\mathrm{CHOP}$ expression. The dependence of these effects on Them 2 expression was evidenced by the absence of changes in the hepatic activities of PERK, IRE1 $\alpha$, eIF2 $\alpha$, and CHOP in compound A1-treated Them $2^{-/}$mice following tunicamycin administration (Supplemental Figure 4). When taken together, these observations in mouse models support a role for a Them $2 / \mathrm{PC}-\mathrm{TP}$ complex in the induction as well as the resolution of ER stress. 
A

\begin{tabular}{|c|c|}
\hline A & ${\text { Them } 2^{+/+} \text {Them }^{-/}}^{-1}$ \\
\hline nicamycin, 1 нg/ml: & -+-+ \\
\hline p-PERK (T980) & 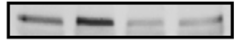 \\
\hline$p-I R E 1 \alpha(S 724)$ & $=-$ \\
\hline $\mathrm{CHOP}$ & $=$ \\
\hline ATF6 & $\Rightarrow$ \\
\hline Them2 & -0 \\
\hline$\beta$-Acti & -2 \\
\hline
\end{tabular}

B

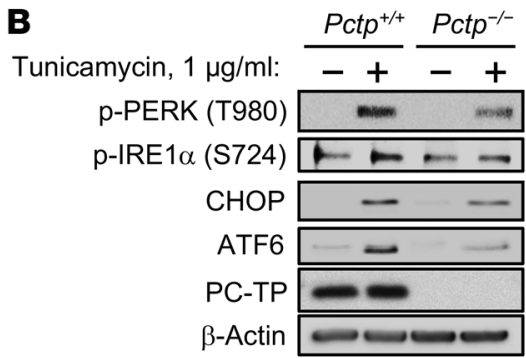

C

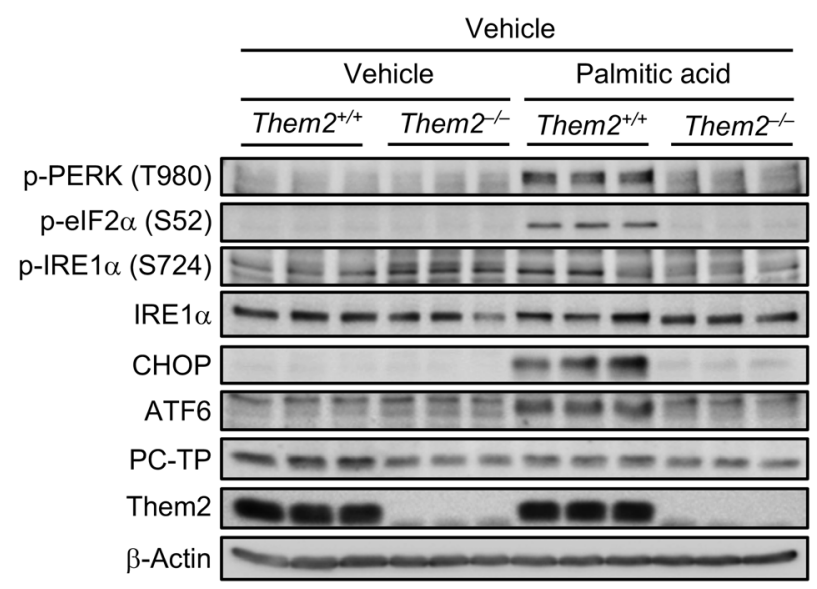

D

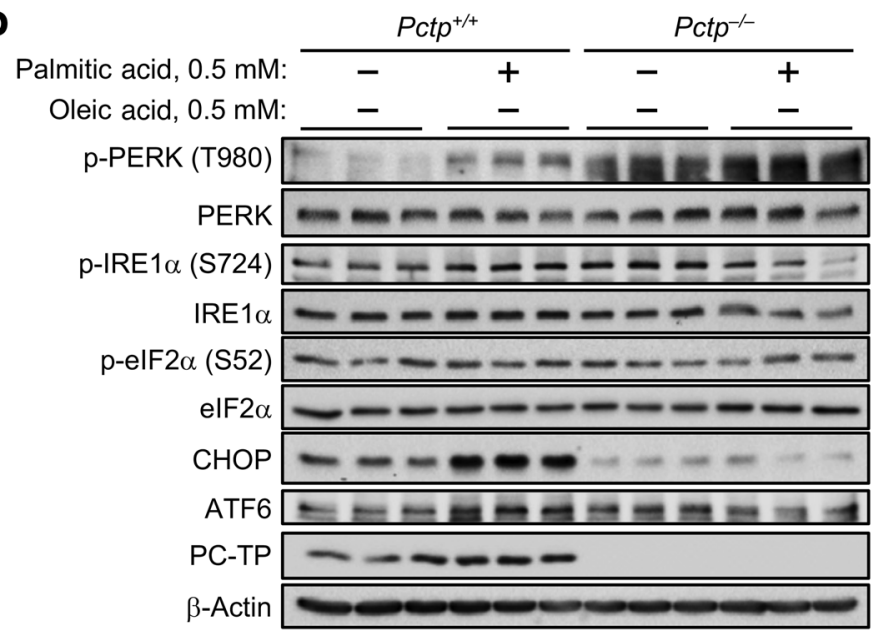

Oleic acid

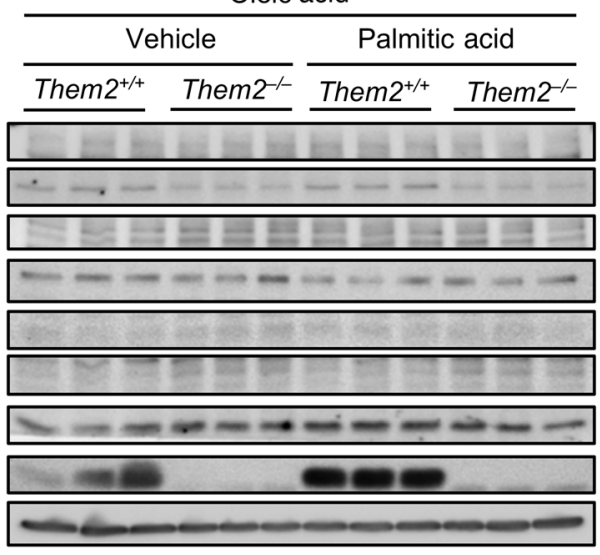

\begin{tabular}{|c|c|c|c|}
\hline \multicolumn{2}{|c|}{ Pctp $^{+/+}$} & \multicolumn{2}{|c|}{$P c t p^{-/-}$} \\
\hline- & + & - & + \\
\hline+ & + & + & + \\
\hline
\end{tabular}

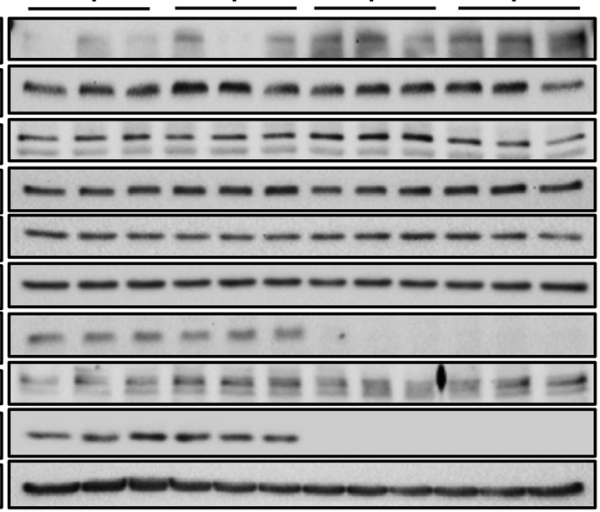

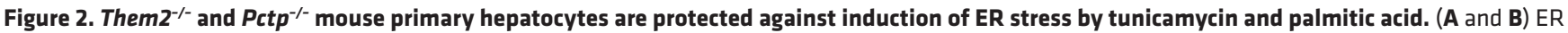

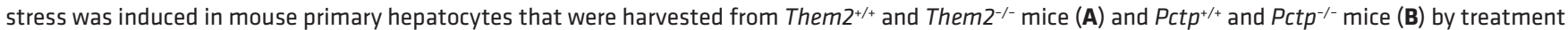
of cells with tunicamycin $(1 \mu \mathrm{g} / \mathrm{ml})$ for 5 hours. (C and $\mathbf{D})$ ER stress was induced in mouse primary hepatocytes harvested from Them2 $2^{+/+}$and Them $2^{-/-}$mice (C) and Pctp ${ }^{+/+}$and Pctp ${ }^{-/-}$mice (D) by treatment of cells with palmitic acid ( $\left.0.5 \mathrm{mM}\right)$ or vehicle (4.8 mM BSA) for 6 hours. Rescue of SFA-induced ER stress was performed by treatment of cells with the monounsaturated NEFA oleic acid $(0.5 \mathrm{mM})$ in the presence of palmitic acid (0.5 mM) or vehicle $(4.8 \mathrm{mM}$ BSA) for 6 hours. Hepatocytes were serum-starved overnight before treatments. Immunoblots are representative of 3 independent experiments.

Cell-autonomous activities of Them 2 and PC-TP in promoting ER stress. In keeping with observations in vivo, tunicamycin-mediated induction of p-PERK, p-IRE1 $\alpha$, CHOP, and ATF6 cleavage in Them $2^{-/-}$and $P c t p^{-/-}$hepatocytes was markedly reduced in comparison with WT hepatocytes (Figure 2, A and B). Under conditions of overnutrition, SFAs play key roles in promoting ER stress, and their effects can be tested directly using primary hepatocytes cultures. Them $2^{-/-}$but not $T h e m 2^{+/+}$hepatocytes were protected against palmitic acid-induced increases in p-PERK, p-eIF $2 \alpha$, CHOP, and cleaved ATF6 expression (Figure 2C). In keeping with our find- ings from compound A1 treatment in mice, the absence of PC-TP similarly activated PERK and suppressed palmitic acid-mediated induction of p-IRE1 $\alpha$, p-eIF $2 \alpha$, and CHOP in primary hepatocytes (Figure 2D). Cotreatment of hepatocytes with oleic acid blocked the induction of ER stress by palmitic acid, irrespective of genotype (Figure 2, C and D).

To exclude the possibility that primary hepatocytes may have adapted to the chronic absence of Them 2 and PC-TP, we used siRNA to silence the expression of these proteins in Hepa1-6 mouse hepatoma cells. Knockdown of Them 2 expression attenu- 
ated all tunicamycin-induced ER stress, but the PC-TP siRNA treatment only reduced p-PERK and cleaved ATF6 abundance following tunicamycin treatment (Supplemental Figure 5A). Knockdown of Them 2 expression suppressed the mRNA transcription of CHOP, whereas PC-TP knockdown reduced both Grp78 and CHOP mRNA expression (Supplemental Figure 5, B and C). Similar protection against ER stress following knockdown of Them 2 in human embryonic kidney (HEK) 293E cells was not reversed by the overexpression of either Acot7 or Acot12 (Supplemental Figure 6), which exhibit different subcellular localization and substrate specificity than Them2 (24).

Them 2 and PC-TP induce ER stress by promoting release of luminal calcium. Reduced ER calcium concentrations are an important trigger of the ER stress response (25). Treatment of hepatocytes with the sarcoplasmic reticulum ATPase $2 b$ (Serca2b) inhibitor thapsigargin increased Them 2 protein expression (Figure 3A) along with p-IRE1 $\alpha$, CHOP, and cleaved ATF6 in WT but not in Them $2^{-/-}$and Pctp ${ }^{-/}$hepatocytes (Figure 3A). Whereas the absence of PC-TP expression also prevented PERK activation, the absence of Them 2 expression reduced this only slightly (Figure 3A). Together these findings suggest that Them2 and PC-TP might promote ER stress by increasing luminal calcium losses. To explore this possibility, we measured the loss of ER calcium into the cytosol using the calcium reporter Fluo4-AM. Because of the high background fluorescence of primary hepatocytes, we used HEK 293E cells: Knockdown of either Them 2 or PC-TP expression led to a $31 \%$ reduction in the efflux of ER calcium following thapsigargin treatment (Figure 3B).

A significant proportion of ER calcium loss into the cytosol following thapsigargin treatment or lipotoxicity occurs as passive diffusion through translocons (26-28). Treatment of cells with anisomycin, a pharmacological inhibitor of translocons (27), reduced the calcium leak from the ER into the cytosol in the scrambled siRNA-treated cells to the same level as in Them 2 and PC-TP siRNA-treated cells (Figure 3C). Presumably because calcium leak via translocons was already reduced, anisomycin treatment did not alter the ER calcium release in Them2 and PC-TP siRNA-treated cells (Figure 3C). These results suggest that Them2 and PC-TP promote ER calcium efflux at least in part through the activity of translocons.

To determine whether Them 2 and PC-TP also affected resting total ER calcium stores, we treated cells with ionomycin, which permeabilizes the ER membrane (29). Whereas knockdown of Them 2 had little effect, knockdown of PC-TP reduced ionomycininduced calcium release (Figure 3D). Whereas the role of membrane fluidity in ionomycin efficacy is not known, the absence of Them2 expression, which increased membrane fluidity, did not appear to reduce ionomycin-mediated calcium release. Potentially explaining these differences in calcium release following ionomycin treatment, Serca2b protein abundance was increased in cells treated with Them 2 siRNA (Figure 3E) but reduced in cells treated with PC-TP siRNA (Figure 3F).

We next eliminated the potential contributions of inositol 1,4,5-triphosphate receptors ( $\mathrm{IP}_{3} \mathrm{Rs}$ ), which, when activated, mediate the efflux of ER calcium, leading to ER stress (30). Unexpectedly, knockdown of Them2 or PC-TP markedly increased $\mathrm{IP}_{3} \mathrm{R}$ expression (Figure $3, \mathrm{E}$ and $\mathrm{F}$ ). The induction of $\mathrm{IP}_{3} \mathrm{R}$ protein was associated with 1.4- and 3.7-fold increases in the mRNA abundance of $I p_{3} r$ subtype 3 ( $I p_{3} r 3$ ) following the knockdown of Them2 and PC-TP expression, respectively (Figure 3, G and H). We used a siRNA targeting $\mathrm{IP}_{3} \mathrm{R} 3$ to neutralize the effects of Them 2 and PC-TP knockdown on $\mathrm{IP}_{3} \mathrm{R}$-mediated calcium release (Figure 3, I and J). Indeed, knockdown of $\mathrm{IP}_{3} \mathrm{R} 3$ reduced ER calcium release (Figure 3K); however, calcium release was further reduced by $21 \%$ and $35 \%$ in response to concomitant knockdown of Them 2 and PC-TP, respectively (Figure 3K). This additional decrement in calcium efflux might have resulted from reduced $\mathrm{IP}_{3}$-mediated activation of $\mathrm{IP}_{3} \mathrm{Rs}$ in the absence of Them 2 and PC-TP. To test this, we treated cells with the protease-activated receptor (PAR) agonist thrombin, which triggers rapid intracellular accumulation of $\mathrm{IP}_{3}$ in response to activation of phospholipase $\mathrm{C}$ (31). However, knockdown of Them2 or PC-TP expression did not impair $\mathrm{IP}_{3}$ mediated ER calcium release (Figure 3L).

We previously demonstrated that knockdown of Them 2 or PCTP expression in HEK 293E cells activates Akt and the mammalian target of rapamycin (mTOR) (16). We found that the regulation of calcium fluxes by Them 2 and PC-TP occurs independently of Akt and mTOR activity, since inhibiting Akt or mTOR activity with GDC-0941 or rapamycin, respectively, did not abolish the effects of Them 2 and PC-TP on ER calcium fluxes (Supplemental Figure 7).

Disruption of calcium homeostasis by palmitic acid requires the expression of Them 2 and PC-TP. Because the incorporation of palmitic acid into the ER membrane induces ER stress in cultured cells by altering ER membrane phospholipid composition and depleting ER calcium $(4,5,32)$, we next tested whether Them 2 and PC-TP might facilitate the depletion of ER calcium in response to SFAs. HEK 293E cells were treated with palmitic acid or vehicle (4.8 mM BSA) for 6 hours prior to the measurement of ER calcium efflux. Indicative of ER calcium depletion, palmitic acid reduced the magnitude $\left(\mathrm{E}_{\max }\right)$ of calcium release in cells treated with scrambled but not Them 2 or PC-TP siRNA following either thapsigargin- or ionomycin-mediated induction of calcium release (Figure 4, A-C). Knockdown of Them 2 expression completely prevented disruption of calcium homeostasis by palmitic acid (Figure 4B). By contrast, palmitic acid treatment increased the duration of calcium release in scrambled and PC-TP siRNA-treated cells (Figure 4, A and C). In scrambled siRNA-treated cells and to a lesser extent in PC-TP siRNA-treated cells, this was associated with a failure to fully restore cytosolic calcium to baseline (Figure 4, A and C). Steady-state cytosolic calcium concentrations were lower by $17 \%$ and $37 \%$ following knockdown of Them 2 and PC-TP expression, respectively (Figure 4D). As anticipated from impaired restoration of baseline cytosolic calcium levels, palmitic acid induced a 1.9-fold increase in cytosolic calcium in scrambled siRNA-treated cells but not in Them 2 siRNA-treated cells (Figure 4D). Knockdown of PC-TP expression also diminished the cytosolic calcium accumulation by $30 \%$ (Figure 4D). These results indicate that a Them2/PC-TP complex is integral to palmitic acid cytotoxicity.

Them 2 and PC-TP reduce ER membrane fluidity. Because ER membrane fluidity regulates the activity of ER calcium channels (33-35), we next assessed whether Them2 and PC-TP may alter ER membrane fluidity, thereby providing a mechanism for the control of calcium homeostasis and ER stress. Loss of Them 2 expression was associated with a $30 \%$ increase in hepatic ER membrane fluidity in comparison with WT littermates as determined by 
A

$\mathrm{Tg}, 0.5 \mu \mathrm{M}: \frac{\text { Them }^{+/ /}}{-\mathbf{+}} \frac{\text { Them2 }^{-/-}}{-\mathbf{+}} \frac{\text { Pctp }^{+/+}}{-\mathbf{+}} \frac{\text { Pctp }^{-/}}{-\mathbf{+}}$

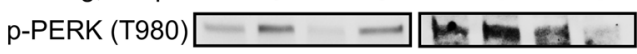
p-IRE1 $\alpha($ S724) $=-$

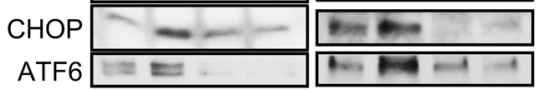
Them2/PC-TP $\beta$-Actin $=2=$ $=-$ $\longrightarrow 2$

C

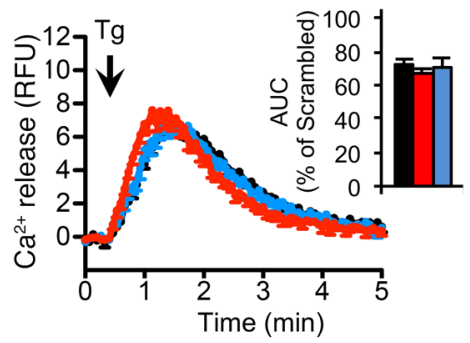

E

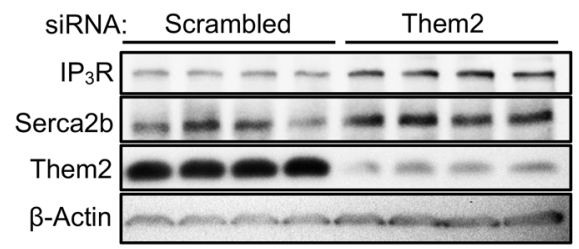

G

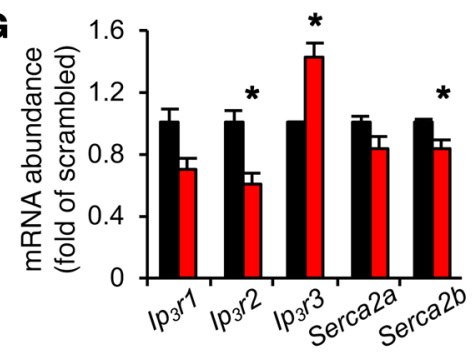

I

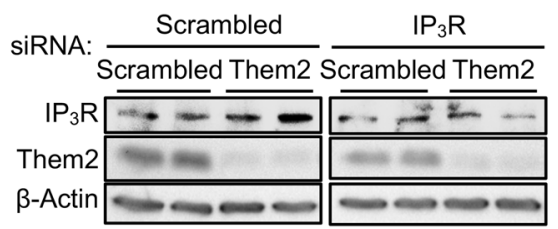

$\mathbf{K}$

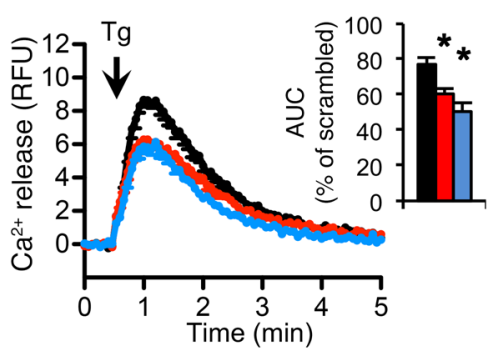

B

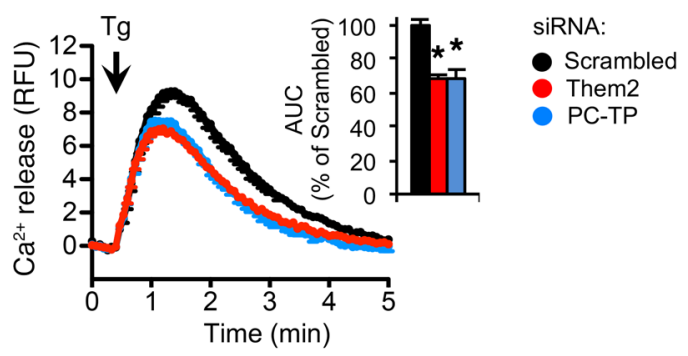

D

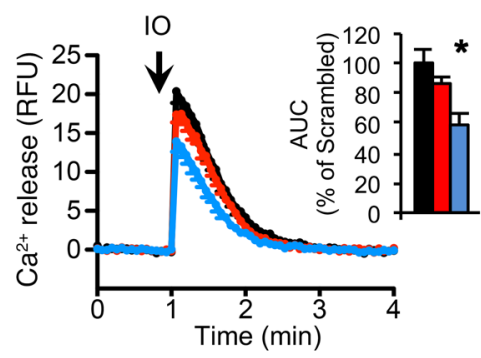

F siRNA: Scrambled PC-TP
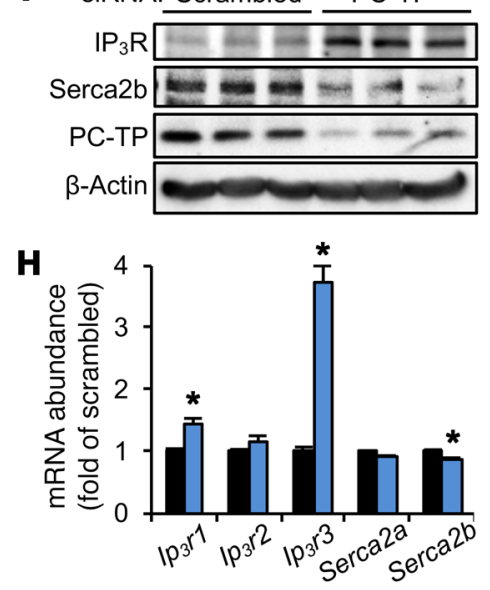

J

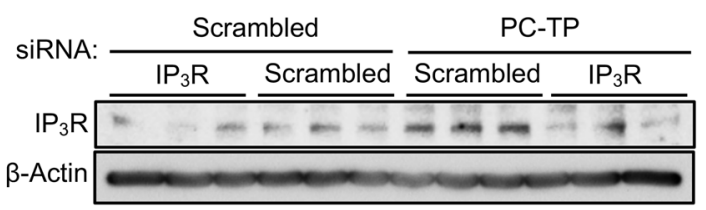

$\mathbf{L}$

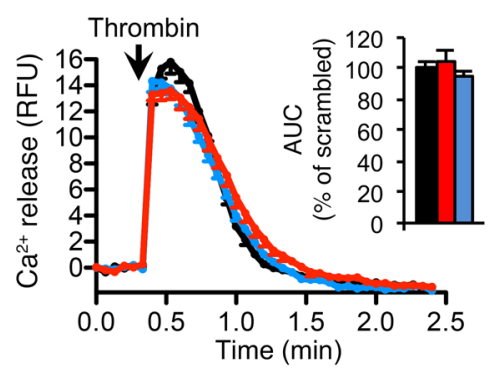


Figure 3. Them2 and PC-TP promote efflux of ER calcium into the cytosol. (A) ER stress induced by $0.5 \mu \mathrm{M}$ thapsigargin ( $\mathrm{Tg}$ ) in mouse primary hepatocytes. Immunoblots are representative of 3 independent experiments. (B) ER calcium release into cytosol induced by $2 \mu \mathrm{M}$ Tg in HEK $293 \mathrm{E}$ cells following knockdown of Them2, PC-TP, or scrambled control. Calcium release into cytosol was measured by Fluo4-AM relative fluorescence units (RFU). Inset barplot displays the AUC. ${ }^{*} P<0.025$ compared with scrambled. (C) HEK $293 \mathrm{E}$ cells were preincubated with the translocon inhibitor anisomycin $(200 \mu \mathrm{M})$ or vehicle $(0.1 \% \mathrm{v} / \mathrm{v}$ DMSO) for 1 hour prior to Tg-induced ER calcium release into the cytosol following knockdown of Them2, PC-TP, or scrambled control. (D) ER calcium release into cytosol induced by $5 \mu \mathrm{M}$ ionomycin (IO) in HEK 293E cells following knockdown of Them2, PC-TP, or scrambled control. ${ }^{*} P<0.025$ compared with scrambled. (E and $\mathbf{F}$ ) Immunoblot analyses of $\mathrm{IP}_{3} \mathrm{R}$ and Serca2b following knockdown of Them2 (E), PC-TP (F), or scrambled control in HEK 293E cells. (G and $\mathbf{H}$ ) mRNA abundance of $I p_{3} r 1, I p_{3} r 2, I p_{3} r 3$, Serca2a, and Serca $b b$ was determined by qPCR analysis following knockdown of Them2 (C), PC-TP (H), or scrambled control in HEK 293 E cells. GAPDH mRNA served as reference. Error bars represent SEM for $n=3 .{ }^{*} P<0.05$ compared with scrambled. (I and J) IP $P_{3} R 3$ expression was knocked down along with Them2 (I), PC-TP (J), or scrambled control. (K) Tg-induced ER calcium release into cytosol following co-knockdown of IP $R 3$ in HEK 293 E cells. AUC was normalized to scrambled control from $B$. ${ }^{*} P<0.025$ compared with scrambled $+I_{3} R 3$ siRNA. (L) IP - -mediated ER calcium release into cytosol was induced in HEK $293 \mathrm{E}$ cells by $40 \mu \mathrm{M}$ thrombin. Calcium release curves represent 6-9 independent experiments. Statistical significance was determined by Student's $t$ test adjusted by Bonferroni correction.

increased pyrenedecanoic acid (PDA) excimer-to-monomer ratios (Figure 5A). Despite a trend toward increased fluidity in the ER membranes isolated from livers of high-fat diet-fed Them $2^{-/-}$mice, the differences did not achieve statistical significance, potentially because of the high saturated fatty acid content of the high-fat diet and the biological variability in response to the diet, as well as the presence of additional cell types within the liver. For these reasons, we further tested the role of Them 2 and PC-TP in membrane fluidity using cell culture systems in which the experimental conditions could be more tightly controlled.

Palmitic acid treatment of cultured cells increases the abundance of membrane phospholipids with saturated and monounsaturated fatty acyl chains while reducing the relative abundance of polyunsaturated fatty acyl chains with 4 or more double bonds (33). Indeed, we observed that palmitic acid treatment of HEK 293E cells reduced ER membrane fluidity 2.4-fold, but these changes were abrogated by knockdown of either Them 2 or PC-TP (Figure 5B). These findings were validated using diphenylhexatriene $(\mathrm{DPH})$ polarization anisotropy to measure membrane fluidity of the ER fractions purified from Them $2^{-/-}$and $\mathrm{Pctp}^{-/-}$mouse primary hepatocytes (Figure 5C). Consistent with effects of Them2 and PC-TP on membrane fluidity, genotype-dependent differences in DPH polarization were eliminated by increasing temperatures (Figure 5C). These results demonstrate that Them 2 and PC-TP regulate ER membrane fluidity, providing a mechanistic connection to ER calcium homeostasis (4).

Them 2 and PC-TP regulate hepatic ER phospholipid composition. To delineate the mechanisms underlying the regulation of ER membrane fluidity by Them 2 and PC-TP, we assessed the regulation of lipid channeling into ER by Them 2 and PC-TP using radiolabeled palmitic acid and oleic acid tracers. Consistent with protection against palmitate-induced ER stress and ER calcium loss, trafficking of radiolabeled palmitic acid into the ER was reduced by $24 \%$ and $26 \%$ in Them $^{-/-}$and $\mathrm{Pctp}^{-/-}$hepatocytes, respectively (Figure 6, A and B). Suggestive of selective targeting of palmitic acid to the ER by Them2/PC-TP, the subcellular localization of radiolabeled oleic acid remained unchanged in the absence of Them 2 and PC-TP (Figure 6, A and B).

Membrane phospholipids with polyunsaturated fatty acyl chains with 4 or more double bonds have been associated with increased membrane fluidity (34-36). We investigated the influence of Them2 and PC-TP on the composition of the major ER membrane phospholipids, phosphatidylcholines (PCs) and phosphatidylethanolamines (PEs), by mass spectrometry analyses (Figure 6, C-H). We observed increased proportions of polyunsaturated PC molecular species (36:4, 38:6, and 40:6) in hepatic ER membrane fractions from highfat-fed Them $2^{-/-}$mice compared with livers of WT mice (Figure 6C). There was a parallel trend toward greater polyunsaturated PE molecular species (38:4 and 38:6) in Them $2^{-/-}$mice (Figure 6D). Similarly in high-fat-fed $\mathrm{Pctp}^{-/}$mice, we observed greater proportions of polyunsaturated $\mathrm{PC}(36: 4,38: 5)$ (Figure 6F) and $\mathrm{PE}(38: 4,38: 5$, and 38:6) (Figure 6G) molecular species compared with those in WT littermates. Consistent with improved ER membrane fluidity in the setting of high-fat diet, the overall abundance of ER phospholipids that contained fatty acids with 4 or more double bonds was elevated by $17 \%$ and $47 \%$ in the livers of Them $2^{-/-}$and $\mathrm{Pctp}^{-/-}$mice, respectively, compared with WT littermates (Figure 6, E and H).

Elevated hepatic ER membrane $\mathrm{PC} / \mathrm{PE}$ ratios due to increased expression of Pemt, which catalyzes the conversion of PE into PC, have been mechanistically linked to increased ER stress due to impaired Serca2b function (37). We therefore tested the influence of Them 2 and PC-TP expression on the hepatic ER membrane $\mathrm{PC} / \mathrm{PE}$ ratio in high-fat-fed mice. Compared with chow diet-fed littermates, ER membrane PC/PE ratios were increased in the livers of high-fat-fed Pctp ${ }^{+/+}$but not of Pctp ${ }^{-/}$, Them $2^{+/+}$, or Them2 $2^{-/-}$ mice (Supplemental Figure 8, A and B). The mRNA abundance of Pemt was unchanged in response to high-fat feeding of Them $2^{+/+}$ and Them $2^{-/}$mice (Supplemental Figure 8C) and was equally increased in the livers of high-fat-fed $P c t p^{+/+}$and $P c t p^{-/-}$mice. This suggests that different $\mathrm{PC} / \mathrm{PE}$ ratios observed in ER membranes from livers of high-fat-fed $P c t p^{+/+}$and $P c t p^{-/-}$were not attributable to changes in Pemt expression (Supplemental Figure 8D). Pcyt1a, encoding a phosphate cytidylyltransferase that regulates the synthesis of PCs from diacylglycerols and choline, was upregulated in both $\mathrm{Pctp}^{+/+}$and Them $2^{+/+}$mice in response to high-fat feeding. Although the absence of Them 2 or PC-TP expression blunted the induction of Pcyt1a, decreased $\mathrm{PC} / \mathrm{PE}$ ratios were observed only in Pctp ${ }^{-/-}$mice (Supplemental Figure 8, C and D).

Them 2 and PC-TP do not promote the hepatic accumulation of lipotoxic molecules. We previously demonstrated that Them2-1mice exhibit increased hepatic concentrations of fatty acyl-CoAs and reduced hepatic NEFAs (15). This suggests that reduced ER stress in these mice may have been the result of reduced accumulation of lipotoxic molecules. To explore this possibility, we measured other lipotoxic intermediates and found that the absence of Them 2 or PC-TP did not reduce the abundance of free cholesterol (Supplemental Figure 9, A and B) or ceramides (Supplemental Figure 9, C and D) in livers of high-fat-fed mice. In the setting of tunicamycin-induced ER stress, there were only trends toward 
A

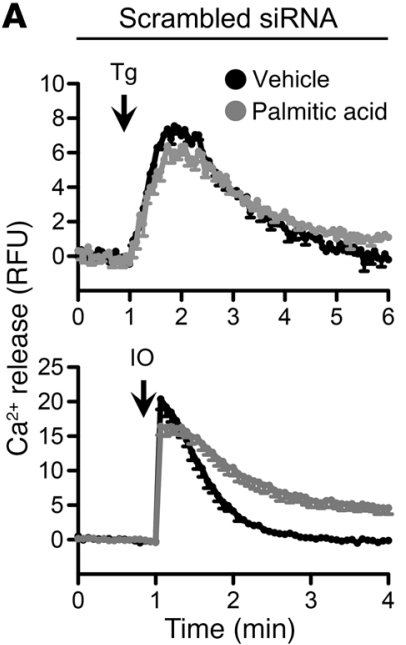

B

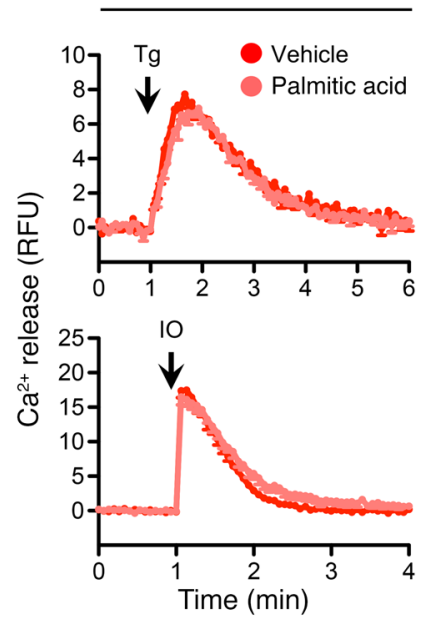

C

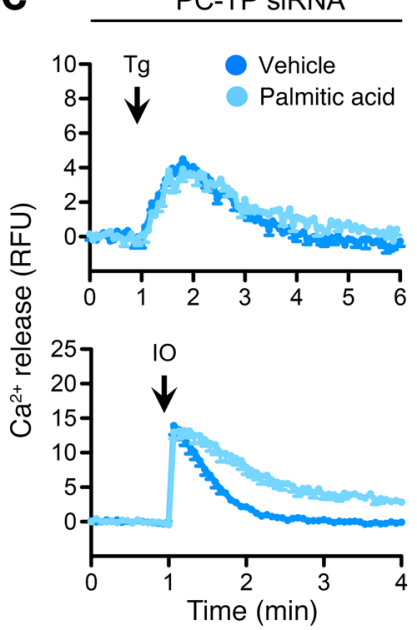

D

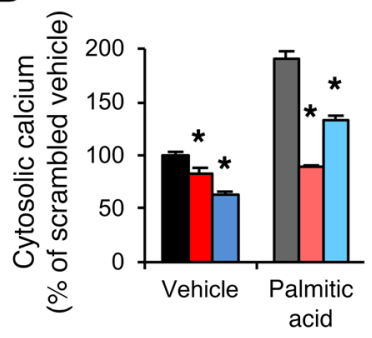

Figure 4. Them2 and PC-TP enable palmitic acid-induced calcium efflux from the ER. (A-C) Serum-starved HEK $293 E$ cells were treated with palmitic acid ( $0.5 \mathrm{mM}$ ) or vehicle (4.8 mM BSA) for 6 hours following knockdown of scrambled control (black/gray) (A), Them2 (red/pink) (B), or PC-TP (blue/aqua) (C). Calcium release into the cytosol was measured as a function of the fluorescence intensity of the cytosolic calcium indicator Fluo4-AM following the induction of ER calcium release by thapsigargin (top panels; Tg, $2 \mu \mathrm{M}$ ) or ionomycin (bottom panels; IO, $5 \mu \mathrm{M}$ ). (D) Steady-state cytosolic calcium levels in HEK 293 E cells following treatment with palmitic acid $(0.5 \mathrm{mM})$ or vehicle $(4.8 \mathrm{mM} \mathrm{BSA})$ for 6 hours. Cells were treated with Them2, PC-TP, or scrambled control siRNA for 48 hours and serum-starved overnight before palmitic acid treatment. ${ }^{*} P<0.025 \mathrm{vs}$. scrambled. Time-dependent calcium release curves and cytosolic calcium measurements represent 6-9 independent experiments. Error bars represent SEM. Statistical significance was determined by Student's $t$ test adjusted by Bonferroni correction.

elevated hepatic NEFA concentrations in the livers of WT mice but not in Them $2^{-/-}$and Pctp ${ }^{-/}$mice (Figure 1, I and L). We performed additional experiments in order to test whether Them 2 and PC-TP promoted ER stress by increasing hepatic uptake of NEFAs. The absence of Them 2 or PC-TP expression was not associated with significant changes in plasma NEFA concentrations in response to tunicamycin (Supplemental Figure 10A). Although tunicamycin administration tended to increase NEFA uptake rate in WT hepatocytes, the absence of Them 2 or PC-TP expression did not reduce hepatic fatty acid uptake, irrespective of tunicamycin treatment (Supplemental Figure 10, B and C). Considering that the absence of Them2 expression does not affect rates of hepatic triglyceride secretion (15), these results argue against a role for hepatic accumulation of lipotoxic intermediates in the regulation of ER calcium homeostasis by Them 2 and PC-TP.

Reduced fatty acid oxidation in the absence of Them 2 or PCTP expression could also have influenced ER stress by decreasing the production of mitochondrial reactive oxygen species (ROS) $(19,38)$. To explore this possibility, we measured hydrogen peroxide concentrations in the mouse livers (39). Although hydrogen peroxide accumulation was reduced by $27 \%$ in high-fat-fed Pctp ${ }^{-1-}$ mice, the absence of Them 2 expression had no effect, irrespective of diet (Supplemental Figure 11, A and B). Taken together, these findings argue against a mechanism whereby Them 2 and PC-TP impair ER calcium balance by increasing ROS.

Them2 and PC-TP activate calcium/calmodulin-dependent protein kinase II. Cytosolic calcium-dependent activation of calcium/calmodulin-dependent protein kinase II (CaMKII) regulates fasting-induced gluconeogenesis (40) and inhibits insulin signaling (41). Because both Them $2^{-/-}$and $P c t p^{-/-}$mice exhibit decreased hepatic glucose production and improved insulin sensitivity, we next investigated whether the regulation of cytosolic calcium homeostasis by Them 2 and PC-TP may represent a mechanism in addition to ER stress for their regulation of gluconeogenesis and insulin signaling. Knockdown of Them2 or PC-TP expression inhibited the phosphorylation of CaMKII (Figure 7A). This occurred in a calcium-dependent manner because the incubation of cells with Bapta-AM, a calcium chelator, negated the effects of Them 2 and PC-TP expression (Figure 7A). Treatment of cells with anisomycin, which blocks Them2- and PC-TP-mediated leakage of ER calcium into the cytosol (Figure 3C), also inhibited Them2- and PC-TP-mediated activation of CaMKII (Figure 7B). The absence of Them 2 expression was also associated with $80 \%$ reduction in the activation of CaMKII in the livers of high-fat-fed mice (Figure 7C and Supplemental Figure 1A), suggesting that reduced cytosolic calcium abundance represents a mechanism for the decrease in hepatic gluconeogenesis and improved insulin sensitivity observed in mice lacking Them 2 expression (15).

\section{Discussion}

This study has identified a key regulatory function for the Them2/ PC-TP complex in the control of ER membrane fluidity, which in turn controls ER calcium homeostasis. Our results support a model whereby in the setting of overnutrition, the incorporation of SFAs into the ER reduces membrane fluidity and promotes ER calcium loss by translocon-mediated diffusion, leading to ER stress and ultimately reduced insulin signaling and increased hepatic glucose production (Figure 7D). The data in cell culture systems reveal the central role for Them 2 in this regulatory mechanism because its expression is required for the SFA-mediated disruption of ER and cytosolic calcium homeostasis. An activating role of PCTP was evidenced by the observation that knockdown of PC-TP expression reduced, but did not eliminate, the loss of ER calcium or its accumulation in the cytosol in response to SFAs (Figure 7D). 
A

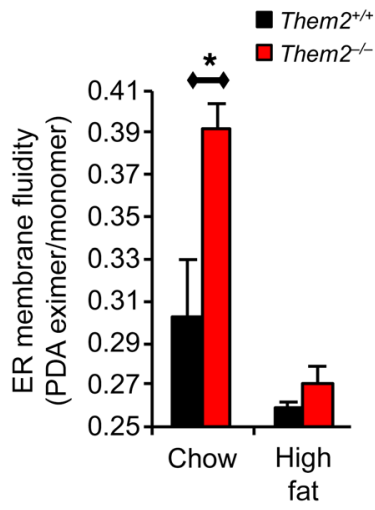

B

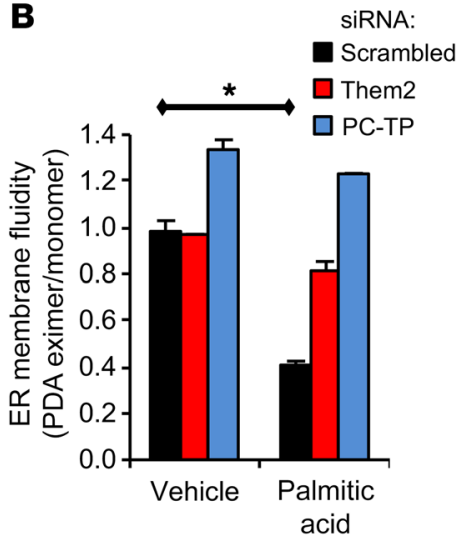

C

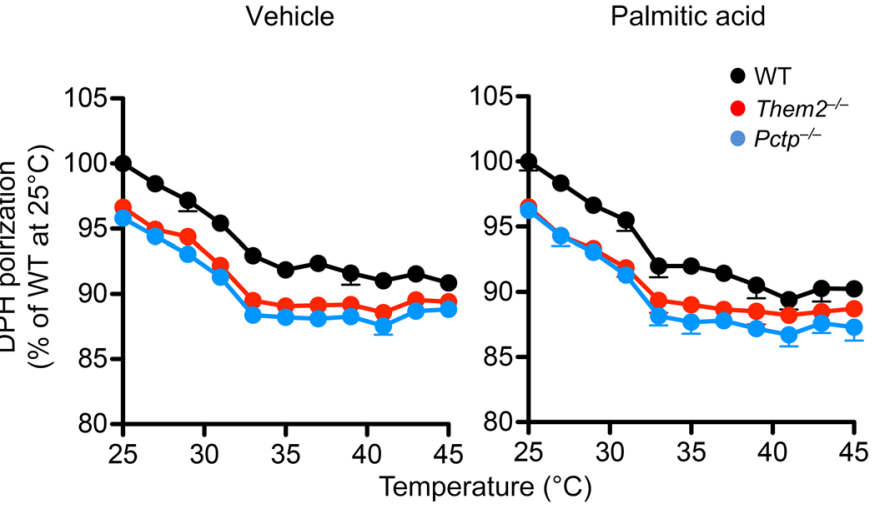

Figure 5. Them2 and PC-TP decrease ER membrane fluidity. (A) ER membrane fluidity as measured by the excimer-to-monomer ratio of pyrenedecanoic acid (PDA). ER microsomes were purified from the livers of chow- or high-fat diet-fed Them2 $2^{+/+}(n=4)$ and Them2 $2^{-/-}(n=4)$ mice following 6 -hour food restriction. Error bars represent SEM. ${ }^{*} P<0.05$ vs. Them $2^{+/+}$mice. (B) ER membrane fluidity of HEK $293 E$ cells as a function of excimer-to-monomer ratio of PDA. Cells were treated with siRNA against Them2, PC-TP, or scrambled control, serum-starved overnight, and incubated with palmitic acid ( $0.5 \mathrm{mM}$ ) or vehicle (4.8 mM BSA) for 6 hours prior to purification of ER fractions. Error bars represent SEM for the triplicate. ${ }^{*} P<0.025$ vs. vehicle. (C) ER membrane fluidity of mouse primary hepatocytes as determined by the inverse correlation of diphenylhexatriene (DPH) polarization anisotropy whereby decreased DPH polarization indicates increased membrane fluidity. Cells were serum-starved overnight and treated with palmitic acid ( 0.5 mM) (right) or vehicle (4.8 mM BSA) (left) for 6 hours. Error bars represent SEM for the triplicate. Statistical significance was determined by Student's $t$ test adjusted by Bonferroni correction.

We previously demonstrated that, under conditions simulating starvation, the Them2/PC-TP complex promotes fatty acid oxidation, apparently by hydrolyzing fatty acyl-CoAs that are in turn esterified by ACSL1 for CPT1-mediated mitochondrial uptake (19). However, when SFAs are present in excess, such as in the livers of high-fat-fed mice (7, 8), ACSL1 also traffics fatty acids to the glycerolipid synthetic pathway (10). Under these conditions, our current findings indicate that the Them2/PC-TP complex facilitates the trafficking of SFAs into the synthesis of glycerolipids, including phospholipids (Figure 7D). By this molecular mechanism, high-fat diet-induced increases in the acyl chain saturation of ER membrane phospholipids reduce membrane fluidity and alter calcium channel activity (34-36), leading to the activation of ER stress and gluconeogenic pathways $(5,40)$.

In addition to promoting chronic ER stress in response to high-fat feeding, Them 2 and PC-TP also contributed to acute ER stress induced by tunicamycin or refeeding. Because tunicamycin-induced ER stress is largely resolved by an upregulation of phospholipid synthesis that leads to membrane expansion (42), reduced trafficking of SFAs as a preferred Them 2 substrate into the glycerolipid synthetic pathway most likely explained the accelerated resolution of ER stress in the absence of Them 2 or PC-TP. The same mechanism would also explain enhanced activation of IRE1 $\alpha$ following refeeding of Them $2^{-/-}$and $P c t p^{-/}$mice: IRE1 $\alpha$ improves protein folding capacity by promoting ER membrane expansion (43). Under the current experimental conditions, tunicamycin treatment did not appreciably alter either glucose tolerance or insulin sensitivity in WT mice. Therefore, the pathophysiological importance of Them 2 and PC-TP in glucose homeostasis in response to tunicamycin-induced ER stress remains unclear.

Our data further indicate that the Them2/PC-TP complex controls ER calcium homeostasis by regulating the activity of translocons, which form pores in the ER membrane that mediate the passive movement of calcium from the ER into cytosol (44), as opposed to active efflux via $\mathrm{IP}_{3}$ Rs. Although other calcium transport proteins, such as ryanodine receptors and presenilins, could have contributed to the leakage of ER calcium, this seems unlikely because ryanodine receptor family proteins primarily regulate muscle contraction, and hepatocytes only express a truncated form of type 1 ryanodine receptor that amplifies $\mathrm{IP}_{3}$-mediated ER calcium release (45). It is also unlikely that Them 2 and PC-TP increase the passive calcium leakage mediated by presenilins (29), because knockdown of presenilin-1 not only fails to protect against ER stress, but rather promotes it in cell culture systems (46).

Calcium transport against a concentration gradient into the ER requires ATP-dependent active transport by Serca channels. In prior studies, we demonstrated that the hepatocytes cultured from Them $^{-/-}$and $\mathrm{Pctp}^{-/-}$mice exhibited reduced rates of fatty acid oxidation (19). Decreased AMPK phosphorylation likely contributed to the observed reductions in fatty acid oxidation, which were accompanied by increased ADP/ATP ratios. Although the reduced available cellular energy for calcium transport by Serca might have been expected to sensitize hepatocytes to the induction of ER stress, we instead observed that the cells lacking Them2 and PC-TP were protected.

The most likely mechanism by which the Them2/PC-TP complex controls calcium diffusion through translocons is by regulating the fluidity of the ER membrane. ER membranes isolated from livers of Them $2^{-/-}$and Pctp $^{-/-}$mice were enriched with unsaturated membrane phospholipids containing fatty acyl chains with 4 or more double bonds, which markedly increase membrane fluidity (36). Our results further support that the changes in the abundance of ER phospholipids must depend on the activities of enzymes that control fatty acid metabolism (e.g., acyl-CoA synthetases, thioesterases, and desaturases), as opposed to differences in the fatty acid compositions of chow and high-fat diets, which do not contain substantial contents of fatty acids containing 4 or more double bonds. Changes in the ER membrane fluidity have also been 

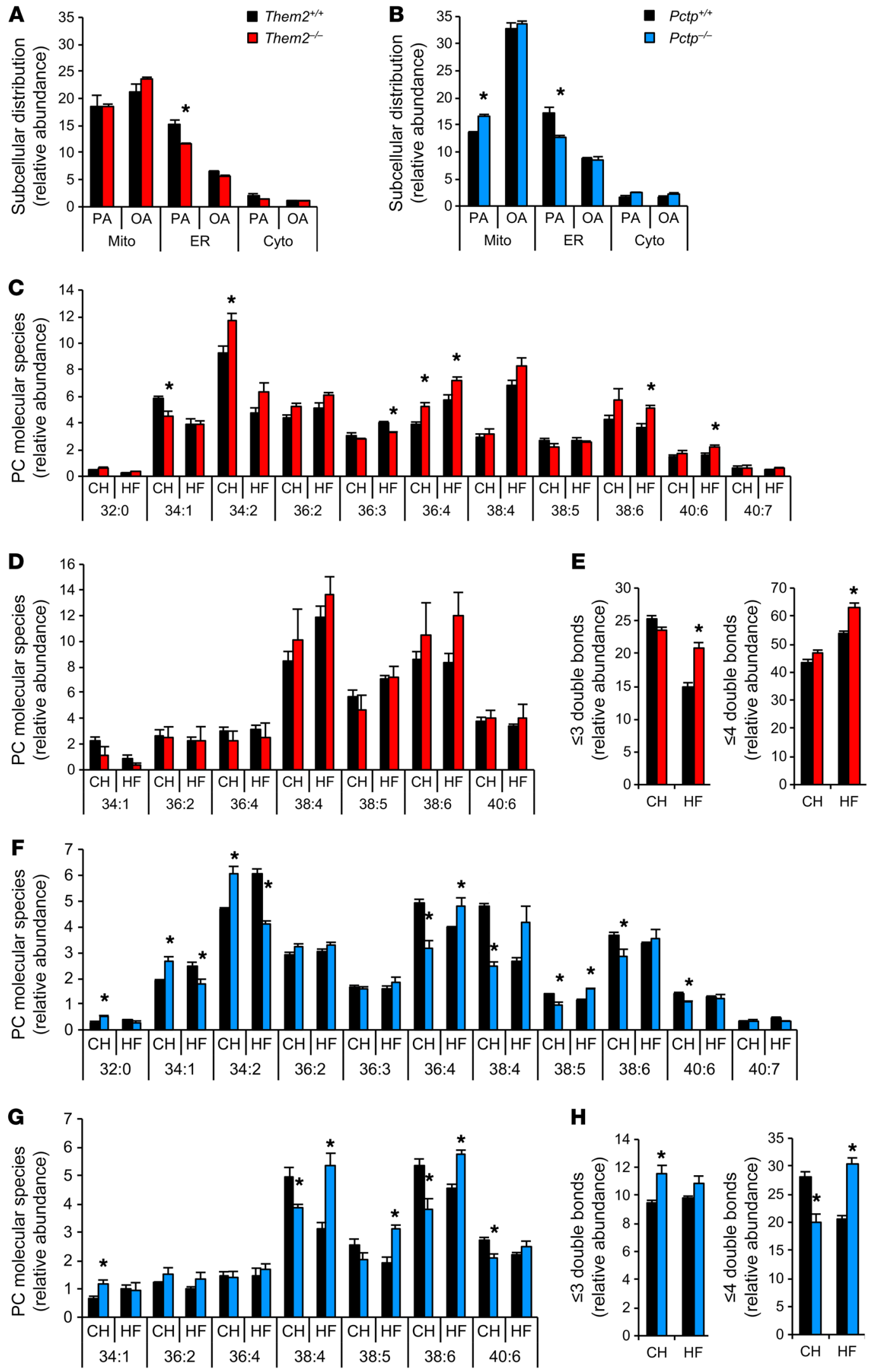
Figure 6. Them2 and PC-TP traffic saturated fatty acids into the ER and reduce fatty acyl chain unsaturation of ER membrane phospholipids in the setting of high-fat diet. (A and $\mathbf{B}$ ) Influence of Them2 (A) and PC-TP (B) on the subcellular distribution of palmitic acid (PA) and oleic acid (OA) Mouse primary hepatocytes were treated with $\left[9,10-{ }^{3} \mathrm{H}\right]$ palmitic acid $(500$ $\mu \mathrm{M}, 10 \mu \mathrm{Ci} / \mathrm{mmol})$ or $\left[9,10-{ }^{3} \mathrm{H}\right]$ oleic acid $(500 \mu \mathrm{M}, 10 \mu \mathrm{Ci} / \mathrm{mmol})$ for 1 hour. Radioisotope distributions to mitochondria (Mito), ER, and cytosol (Cyto) were normalized for subcellular fraction protein amounts, as well as total cellular uptake. (C-H) Four-week-old mice were fed chow or high-fat diet for 8 weeks, and livers were harvested following 6 hours of food restriction. Lipids were extracted from purified ER microsomes and subjected to mass spectrometry analysis. (C and D) Hepatic ER membrane PC (C) and PE (D)

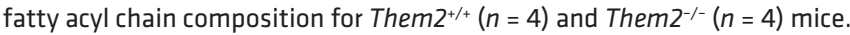
(E) Phospholipid molecular species from $\mathbf{C}$ and $\mathbf{D}$ were divided into 2 groups by fatty acyl chain saturation: $\leq 3$ double bonds (left) or $\geq 4$ double bonds (right). (F and $\mathbf{G})$ Hepatic ER membrane PC (F) and PE (G) fatty acyl chain composition for $\mathrm{Pctp}^{+/+}(n=4)$ and $\operatorname{Pctp}^{-/-}(n=4)$ mice. (H) Phospholipid molecular species from $\mathbf{F}$ and $\mathbf{G}$ were divided into 2 groups as in $\mathbf{C}$. Error bars represent SEM. ${ }^{*} P<0.05$ vs. WT mice. Statistical significance was determined by Student's $t$ test.

demonstrated to modulate Serca2b activity in other experimental settings $(35,47)$. Impaired Serca2b activity has been attributed to increases in PC/PE ratios of the hepatic ER membranes (37). In that study a $52 \%$ increase in the ER PC/PE ratio was mechanistically linked to ER stress in livers of chow-fed $o b / o b$ mice. A likely explanation was increased rates of conversion of PE to PC due to upregulation of Pemt (37). In the current study, high-fat feeding increased the ER PC/PE ratios only modestly (16\%) in the livers of $\mathrm{Pctp}^{+/+}$mice, and had no effect in $\mathrm{Pctp}^{-/}$, Them2 ${ }^{+/+}$, and Them2 $2^{-/}$ mice. Expression of Pcyt1a, and not Pemt, was more consistently upregulated in livers of both $\mathrm{Them}^{+/+}$and $\mathrm{Pctp}^{+/+}$mice, suggesting that the Kennedy pathway (48) played a more central role in PC synthesis in response to high-fat diet-induced ER stress.

The role of calcium-dependent signaling pathways in the activation of hepatic glucose production has been well characterized (49). These effects likely contributed to the reduced hepatic gluconeogenesis observed in Them $2^{--}$and Pctp ${ }^{-/}$mice $(15,17)$, as well as in primary hepatocyte cultures (19). In the absence of either protein, reduced efflux of ER calcium into the cytosol was associated with reduced activation of CaMKII. In addition, the suppression of ER stress in the absence of Them2 or PC-TP expression prevented the downstream activation of $\mathrm{CHOP}$, and this presumably contributed to improved hepatic insulin sensitivity observed in high-fatfed Them $2^{-/}$and Pctp ${ }^{-/}$mice $(15,17)$.

We have proposed that the Them2/PC-TP complex plays an important role in the selection and trafficking of fatty acids into oxidative or glycerolipid biosynthetic pathways (20). Our current findings provide a plausible mechanism for our previous observations in cultured hepatocytes that, under physiological conditions, PC-TP and Them2 interactions increase in response to fasting and function to promote hepatic glucose production (19). Under the current experimental conditions simulating hepatic ER stress, the activation of calcium-dependent hepatic glucose production by the interactions of PC-TP and Them2 is likely to reflect a maladaptive manifestation of this response: The same complex promotes ER stress by channeling excess SFAs to the phospholipids of the ER membrane. Reduced membrane fluidity increases calcium leak via translocons leading to increases in hepatic glucose production and insulin resistance. These findings further establish the Them2/PC-TP complex as an attractive target for the management of hepatic steatosis and insulin resistance.

\section{Methods}

Materials. Media and supplies for cell culture experiments were purchased from Invitrogen. Rapamycin and GDC-0941 were from EMD Chemicals and Chemdea, respectively. Tunicamycin, thapsigargin, anisomycin, thrombin, palmitic acid, oleic acid, and fatty acid-free BSA were from Sigma-Aldrich. Polyclonal antibodies against PC-TP and Them2 were as previously described (13). Antibodies against IRE1 $\alpha$ (3294), eIF2 $\alpha$ (9722), p-PERK (T980) (3179), PERK (3192), and Serca2b (4388) were from Cell Signaling Technology. Antibodies against Grp78 (G8918) and $\beta$-actin (A5441) were from SigmaAldrich. Antibodies against p-CAMKII (T286) and p-eIF2 $\alpha$ (S52) were obtained from Novus Biologicals (NB110-96869) and Enzo Life Sciences (ADI-KAP-CP131), respectively. Bapta-AM and p-IRE1 $\alpha$ (S724) antibody (ab48187) was from Abcam. Antibodies against CAMKII (sc-13082), CHOP (sc-7351), normal rabbit IgG (sc-2027), and Acot7 (sc-376808) were obtained from Santa Cruz Biotechnology. An antibody that reacts with all $3 \mathrm{IP}_{3} \mathrm{R}$ isoforms was from Millipore (07-1210). An antibody against cleaved ATF6 $\alpha$ was provided by Ann-Hwee Lee (Weill Cornell Medical College) (50). Synthetic phospholipid standards for mass spectrometry were obtained from Avanti Polar Lipids. 1,6-Diphenyl-hexa-1,3,5-triene (DPH) was from Sigma-Aldrich. [9,10$\left.{ }^{3} \mathrm{H}\right]$ Palmitic acid and $\left[9,10^{-3} \mathrm{H}\right]$ oleic acid were from Perkin-Elmer.

Animals and experimental induction of ER stress. Male Pctp ${ }^{+/+}$and Pctp $/$ mice and male Them2 $2^{+/+}$and Them2 $2^{--}$mice were as previously described $(15,51)$. Mice were housed in a standard 12-hour alternate light/dark cycle and fed a standard rodent diet 5001 (LabDiets) with free access to drinking water. For studies of diet-induced ER stress, mice were fed a high-fat diet (60\% kcal; D12492; Research Diets) starting at 4 weeks of age for 8 weeks. Livers were harvested from mice following withdrawal of food, but not water, for 6 hours (9:00 am-3:00 pm), and used immediately or snap-frozen in liquid $\mathrm{N}_{2}$ and stored at $-80^{\circ} \mathrm{C}$. For studies of chemical-induced ER stress, 8-week-old chow diet-fed mice were treated with an i.p. injection of tunicamycin $(0.25 \mathrm{mg} / \mathrm{kg}$ body weight) or vehicle ( $0.1 \% \mathrm{DMSO}$ vol/vol) for 2 consecutive days. Livers were harvested 24 hours after the second injection. ER stress was also induced by fasting-refeeding: After an overnight fast, 8 -week-old mice were provided with free access to chow. Livers were harvested at times ranging up to 5 hours after refeeding. In selected experiments, mice were given compound A1 ( $5 \mathrm{mg} / \mathrm{kg}$ body weight dissolved in $4 \%$ DMSO and $96 \%$ of $6 \%$ hydroxypropyl- $\beta$-cyclodextrin vol/vol) or an equal volume of vehicle i.p. daily for 5 days (17). Tunicamycin was coadministered to mice on day 4 and day 5 of compound A1 injection. Livers were harvested 1 day after the second tunicamycin injection.

Glucose and insulin tolerance tests. Tolerance tests to glucose (GTTs) and insulin (ITTs) were performed at the end of 2-day tunicamycin treatment of mice as described above. Food was removed from cages, leaving mice with free access to water 6 hours before i.p. injection with $1 \mathrm{mg} / \mathrm{g}$ body weight D-glucose $(20 \% \mathrm{wt} / \mathrm{vol})$ for GTTs or $1 \mathrm{U} / \mathrm{kg}$ body weight insulin (HumulinR; Eli Lilly) for ITTs. Plasma glucose concentrations were monitored periodically for up to 120 minutes. Because of variations in body weight loss following tunicamycin administration, GTT and ITT curves were normalized to the basal plasma glucose concentrations, which were measured just before injection of glucose or insulin. 
A

\begin{tabular}{|c|c|c|c|c|c|c|c|}
\hline & \multicolumn{3}{|c|}{ Vehicle } & \multicolumn{3}{|c|}{ Bapta-AM } \\
\hline$\varangle$ & Scrambled & + & - & 二 & + & 二 & - \\
\hline zo & PC-TP & - & + & 二 & - & + & - \\
\hline $\bar{\omega}$ & Them2 & - & - & + & - & 二 & + \\
\hline
\end{tabular}

p-CaMKII (T287) - -

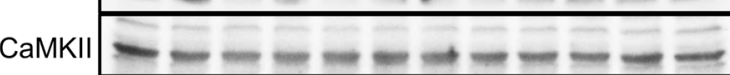

Them2 $=-\mathrm{es}=$

PC-TP $-\sim-\cdots-\cdots-\cdots$

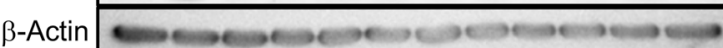

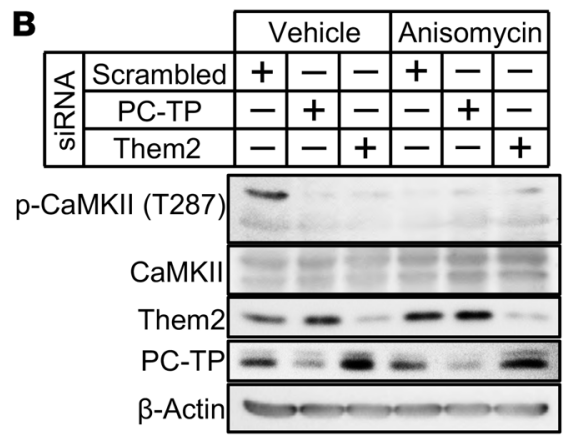

Plasma membrane

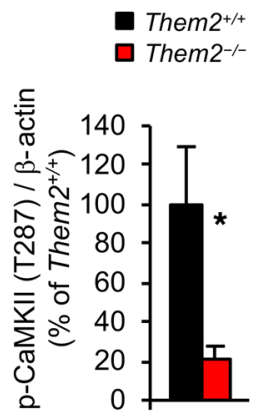

D

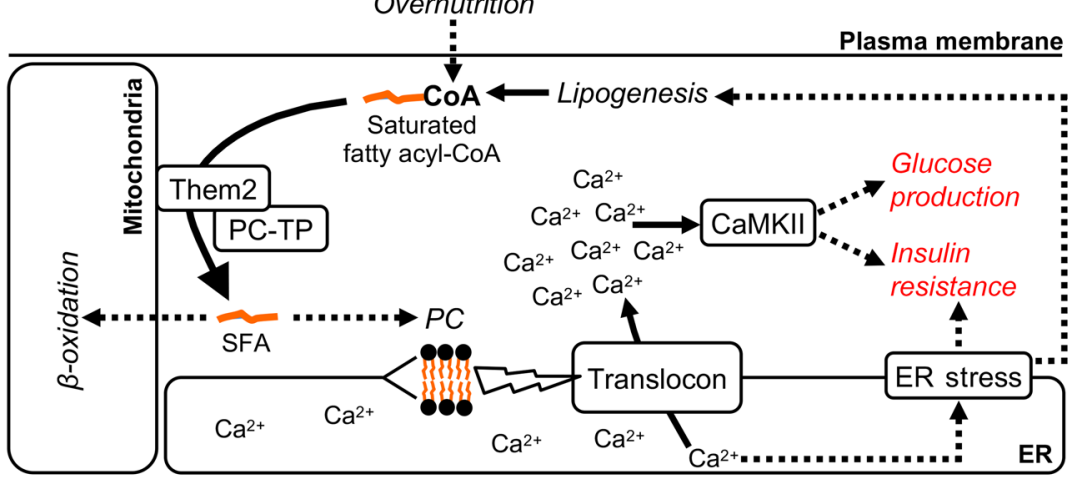

Figure 7. Them2 and PC-TP regulate the activity of CaMKII. (A and B) Influence of Them2 or PC-TP knockdown on CaMKII activation was determined in HEK 293E cells treated with Bapta-AM (5 nM) (A), anisomycin $(200 \mu \mathrm{M})(B)$, or vehicle for 1 hour. Immunoblots represent 3 independent experiments. (C)

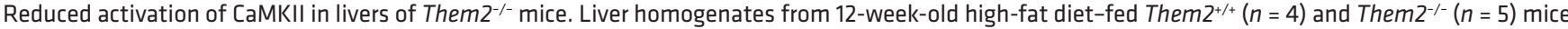
were subjected to immunoblot analyses, and bands were quantified by densitometry and normalized to $\beta$-actin as control. Error bars represent SEM. ${ }^{*} P<0.05$ vs. Them $2^{+/+}$. Statistical significance was determined by Student's $t$ test. (D) Postulated mechanism by which Them2 and PC-TP regulate hepatic glucose homeostasis. In the setting of overnutrition, Them2 and PC-TP are proposed to facilitate the incorporation of SFAs into the ER membrane phospholipid composition, which induces loss of ER calcium into the cytosol via translocons. Increased cytosolic calcium accumulation activates CaMKII, which in turn promotes insulin resistance and enhances hepatic glucose production. Efflux of ER calcium promotes ER stress, which is associated with insulin resistance and de novo lipogenesis.

Liver histology and biochemical analyses. Liver samples were fixed in formalin (Sigma-Aldrich) solution and embedded in paraffin, sectioned to $10 \mu \mathrm{m}$ using a microtome, stained with $\mathrm{H} \& \mathrm{E}$, and visualized using an Eclipse Ti-U microscope equipped with a digital camera (Nikon). Images were standardized for comparison by the inclusion of a bile duct in the visual field. Concentrations of total and free cholesterol, triglycerides, and NEFA were determined in liver and plasma using reagent kits (Wako Chemicals).

Hydrogen peroxide measurement. Hydrogen peroxide accumulation was determined in the livers of mice using a Hydrogen Peroxide Assay Kit (Abcam) according to the manufacturer's specifications. Briefly, livers were homogenized by Dounce homogenizer and deproteinated using $1 \mathrm{M}$ perchloric acid. Hydrogen peroxide concentration was determined by colorimetric absorbance at $570 \mathrm{~nm}$.

Cell culture and transfection. Human embryonic kidney (HEK) 293E (52) and Hepa1-6 (ATCC) cells were cultured in DMEM supplemented with $10 \%$ (vol/vol) FBS (Invitrogen). siRNAs (Ambion) targeting human PC-TP (ID: 121081) and THEM2 (ID: 119801; Ambion) as well as a scramble control (ID: AM4611; Ambion) were used to knock down protein expression (16). A custom Silencer Select siRNA that targets human $\mathrm{IP}_{3} \mathrm{R} 3$ was designed using the sense 5'-UGAGAAGCAGAAGAAGGAGtt-3' and antisense 5'-CUCCUUCUUCUGCUUCUCAcU-3' sequences (Invitrogen, catalog
4390827). cDNAs encoding ACOT7 and ACOT12 (Open Biosystems) were cloned into a pcDNA3 plasmid (Invitrogen) using SmaI and XbaI to release the ACOT7 and ACOT12 cDNAs from the host pCMVSPORT6 plasmid and EcoRV and XbaI to linearize the pcDNA3 plasmid. Cells were transfected with totals of $30 \mathrm{nM}$ siRNA and $0.25 \mathrm{~g} / \mathrm{l}$ plasmid DNA using 0.2\% Lipofectamine 2000 reagent (Invitrogen) according to the manufacturer's protocol. Transfection complexes were prepared in Opti-MEM Reduced Serum Medium (Invitrogen) and added to HEK 293E cells at 50\% confluence in DMEM-10\% FBS. Experiments were conducted 48 hours following transfection after overnight serum starvation.

Mouse primary hepatocytes were isolated and cultured from 6- to 9-week-old mice $(16,18)$. Briefly, mice were anesthetized with an i.p. injection of ketamine (100 mg/kg body weight; Webster Veterinary), xylazine (10 mg/kg body weight; Webster Veterinary), and acepromazine ( $3 \mathrm{mg} / \mathrm{kg}$ body weight; Webster Veterinary). Livers were perfused in situ with $15 \mathrm{ml}$ of liver perfusion media for 4 minutes (Invitrogen) followed by $30 \mathrm{ml}$ of liver digestion media for 8 minutes (Invitrogen) at $37^{\circ} \mathrm{C}$. Digested livers were removed, and the hepatocytes were released into $10 \mathrm{ml}$ each of ice-cold hepatocyte wash media (Invitrogen) by rupturing of Glisson's capsule. Cells were passed through a 70- $\mu \mathrm{m}$ filter (BD Biosciences), pelleted by centrifugation (50 $g$ for 2 minutes), and washed 2 more times before resuspension in cold $\left(4^{\circ} \mathrm{C}\right)$ 
Williams E medium (Invitrogen) containing 10\% FBS, $1 \mu \mathrm{M}$ dexamethasone, and $20 \mathrm{ng} / \mathrm{ml} \mathrm{EGF}$. Hepatocytes were plated on Primaria plates (BD Biosciences) at $80 \%$ confluence. The media were replenished the following day. For the chemical induction of ER stress, cultured hepatocytes were serum-starved overnight and then incubated with $1 \mu \mathrm{g} /$ $\mathrm{ml}$ tunicamycin, $0.5 \mu \mathrm{M}$ thapsigargin, or vehicle (0.1\% DMSO vol/vol) for 5 hours. Alternatively, hepatocytes were serum-starved for 8 hours and then incubated 12 hours with $500 \mu \mathrm{M}$ palmitic acid or oleic acid complexed to BSA (19) or BSA alone in $150 \mathrm{mM} \mathrm{NaCl}$.

Calcium exchange between ER and cytosol. Cellular cytosolic calcium levels were measured as previously described (53). Briefly, cellular and extracellular calcium contents were labeled by loading of HEK 293E cells with $3 \mu \mathrm{M}$ Fluo-4 AM (Invitrogen) in HBSS (Invitrogen) with calcium for 30 minutes at $37^{\circ} \mathrm{C}$. Extracellular calcium was cleared by washing of cells 3 times with calcium-free HBSS. Steady-state cytosolic calcium levels were measured for 1 minute at $23^{\circ} \mathrm{C}$ in calcium-free HBSS. Calcium release from the ER was initiated using thapsigargin, which inhibits Serca2b-mediated calcium reuptake at a concentration of $2 \mu \mathrm{M}$. This concentration was chosen because it exceeds the threshold established for complete inhibition of Serca activity at the highest levels of cellular Serca2 expression (54). Alternatively, ER calcium efflux was induced by activation of $\mathrm{IP}_{3} \mathrm{R}$ channels using $40 \mu \mathrm{M}$ of the human PAR1 agonist thrombin (Sigma-Aldrich). Total ER calcium stores were determined by measurement of ER calcium loss in response to treatment with ionomycin (Abcam), an ionophore that creates calcium-permeable pores (29). Calcium release into cytosol was monitored as time-dependent increase in Fluo-4 AM fluorescence intensity using the SpectraMax M5 microplate reader at excitation and emission wavelengths of 494 and $516 \mathrm{~nm}$, respectively, in calcium-free HBSS. Cytosolic calcium was recorded at the beginning of each experiment in order to establish the background intensity for each cell culture system, and values of calcium release were normalized to the background level. AUC values were calculated using Prism 5 (GraphPad Software).

Membrane fluidity. ER membrane fluidity was determined by 2 independent techniques. The first was to measure changes in the fluorescence polarization of DPH (55). Briefly, ER microsomal fractions were resuspended in a KCl-based buffer $(150 \mathrm{mM}$ $\mathrm{KCl} ; 10 \mathrm{mM}$ HEPES, pH 7.4; 2 mM EGTA) and incubated with 10 $\mu \mathrm{M} \mathrm{DPH}$ at $45^{\circ} \mathrm{C}$ for 30 minutes. Endpoint fluorescence polarization measurements were performed using a Polarstar Omega plate reader (BMG Labtech) at excitation wavelength of $355 \mathrm{~nm}$ and parallel and perpendicular emission wavelengths of $440 \pm 10 \mathrm{~nm}$. The temperature was ramped from $25^{\circ} \mathrm{C}$ to $45^{\circ} \mathrm{C}$ in $2^{\circ} \mathrm{C}$ intervals. The second approach was to measure the formation of pyrenedecanoic acid (PDA) excimers using a Membrane Fluidity Kit (MGT-M0271; Axxora, Enzo Life Sciences). ER microsomes were resuspended in PBS and incubated with $10 \mu \mathrm{M}$ PDA in the presence of $0.08 \%$ Pluronic F127 for 20 minutes at $25^{\circ} \mathrm{C}$. After PDA incorporation into the membranes, microsomes were washed 3 times with PBS to remove excess PDA and resuspended in fresh PBS. Endpoint fluorescence measurements were obtained using a SpectraMax M5 microplate reader (Molecular Devices) at an excitation wavelength of $360 \mathrm{~nm}$. PDA monomer and excimer emissions were detected at 400 and $470 \mathrm{~nm}$, respectively. Microsomal concentrations were titrated to obtain an even distribution of PDA molecules with an excimer-tomonomer ratio of 1 under basal conditions such as chow-fed mouse livers and vehicle-treated cells. Because increased membrane fluidity favors PDA excimer formation, excimer emission intensity was normalized to monomer emission intensity in order to quantify relative membrane fluidity. Microsomal concentrations were determined and normalized based on microsomal protein abundance prior to the analysis of membrane fluidity using DPH and PDA.

Membrane phospholipid compositions. Subcellular fractions enriched with ER were isolated by serial centrifugation at $4^{\circ} \mathrm{C}(56)$. Briefly, 500-mg liver samples were gently homogenized in ice-cold 250-STM buffer (250 mM sucrose; $50 \mathrm{mM}$ Tris- $\mathrm{HCl}, \mathrm{pH}$ 7.4; $5 \mathrm{mM}$ $\mathrm{MgCl}_{2}$ ) using a Dounce Teflon pestle homogenizer (1,000 rpm for 2 minutes). Homogenates were first centrifuged (800 $g$ for $15 \mathrm{~min}$ utes) to pellet unbroken cells and nuclei. Supernatants were centrifuged (6,000 $g$ for 15 minutes) to pellet mitochondrial fractions. After removal of mitochondria, supernatants were centrifuged $(100,000 \mathrm{~g}$ for 1 hour) to pellet ER fractions. ER lipids were extracted by a modified Bligh and Dyer method (57). Briefly, ER pellets were resuspended in $1 \mathrm{ml}$ of PBS, mixed with $2 \mathrm{ml}$ methanol and $1 \mathrm{ml}$ of chloroform, and vortexed for 2 hours at room temperature. After centrifugation (150 $\mathrm{g}$ for 10 minutes), supernatants were mixed with $2 \mathrm{ml}$ of chloroform and $1 \mathrm{ml}$ of $1 \mathrm{M} \mathrm{NaCl}$, then centrifuged (150 $\mathrm{g}$ for 10 minutes) to achieve phase separations. The lower organic phases containing the phospholipids were dried under a stream of $\mathrm{N}_{2}$. For tandem mass spectrometry analyses, dried lipids were reconstituted in chloroform/ methanol $(1: 1 \mathrm{vol} / \mathrm{vol})$ to a final lipid concentration of $5.5 \mathrm{mg} / \mathrm{ml}$. Using a syringe pump, lipids were directly injected into a QTrap 3000 mass spectrometer (Applied Biosystems) at a flow rate of $5 \mathrm{ml} / \mathrm{min}$. To resolve PC molecular species, the mass spectrometer was operated in the positive ion mode (58). The negative ion mode was used (59) to resolve PE molecular species. For both ionization modes, the electrospray ionization energy was $( \pm) 4,000 \mathrm{~V} . \mathrm{N}_{2}$ was used as both the nebulizer and the collision gas. Declustering and collision energies were $70 \mathrm{eV}$ and $35 \mathrm{eV}$, respectively. Q1 and $\mathrm{Q} 3$ were operated in unit mass resolution settings with a step size of $0.5 \mathrm{amu}$. Experimental conditions were optimized using a mixture of synthetic PC (sn-1sn-2 acyl chains; 16:0-18:1, 16:0-18:2, 18:0-18:2) and PE (16:0-18:1 and 16:0-18:2) standards. To identify molecular species of PC and PE, a series of precursor ion scans were performed, with mass-to-charge ratios $(m / z)$ of $184(+)$ and $196(-)$, as these masses correspond to key fragments from the phospholipid head group, respectively. Identified ions were then subjected to product ion scans to determine the sn-1 and sn-2 fatty acyl chain species. To calculate the relative abundance of ER membrane PC and PE species, precursor ion scanning for $(+)$ $m / z 184$ and $(-) m / z 196$, respectively, was completed to specifically target each subset of lipids. The signal at the apex of the peak of interest was divided by the sum total of all peaks identified in each precursor ion scan to calculate that lipid's relative abundance.

Hepatic ceramide concentrations. Hepatic ceramide concentrations were measured by the Metabolomics Platform at the Broad Institute (Cambridge, Massachusetts, USA) as previously described $(60,61)$. Livers were harvested 6 hours after food removal, and the hepatic lipids were extracted from liver homogenates using isopropanol containing $N$-heptadecanoyl-D-erythro-sphingosine (Avanti Polar Lipids) as an internal standard. Lipids were purified by HPLC, and mass spectrometry analyses were performed using electrospray ionization in the positive ion mode using full-scan analysis over $\mathrm{m} / \mathrm{z}$ $200-1,100$ at 70,000 resolution and a $3-\mathrm{Hz}$ data acquisition rate. 
Subcellular distribution of exogenous fatty acids in cultured hepatocytes. The subcellular distribution of exogenous fatty acids following uptake into cultured hepatocytes was essentially as previously described (4). Primary mouse hepatocytes were cultured in 100-mm dishes, serum-starved overnight, and then incubated for 1 hour with $500 \mu \mathrm{M}$ BSA-conjugated palmitic acid or oleic acid, which were radiolabeled using $\left[9,10-{ }^{3} \mathrm{H}\right]$ palmitic acid $(53.7 \mathrm{Ci} /$ mmol) or $\left[9,10-{ }^{3} \mathrm{H}\right]$ oleic acid $(54.5 \mathrm{Ci} / \mathrm{mmol})$, respectively, at a final specific activity of $10 \mu \mathrm{Ci} / \mathrm{mmol}$. Cells were then washed twice with PBS and then subjected to subcellular fractionation of ER, mitochondria, and cytosol by centrifugation, as described above. Radioactivity was determined by suspension of subcellular fractions in Ecoscint $\mathrm{H}$ scintillation solution (National Diagnostics) for quantification using an LS6000IC liquid scintillation counter (Beckman Coulter), and normalized to protein concentration of the respective fraction. To account for changes in the uptake of radiolabeled fatty acids, data were further normalized to total cellular radioactivity before fractionation.

Rates of hepatocellular NEFA uptake. NEFA uptake rates into mouse primary hepatocytes were measured using QBT Fatty Acid Uptake Assay Kit (Molecular Devices) according to the manufacturer's protocol. Cells (20,000 cells per well in 96-well format) were serum-starved for 1 hour and then treated with $1 \mu \mathrm{g} / \mathrm{ml}$ tunicamycin or vehicle $(0.1 \% \mathrm{DMSO}$, vol/vol) for 5 hours prior to the measurement of NEFA uptake according to time-dependent increase in fluorescence intensity using a microplate reader (Molecular Devices) at excitation and emission wavelengths of 485 and $515 \mathrm{~nm}$, respectively. NEFA uptake rates (slopes) were calculated using the linear regression function of Prism 5.

Immunoblot analysis. Immunoblot analyses were as previously described (16). Cells or liver samples were washed with PBS and homogenized in ice-cold RIPA buffer (50 mM Tris- $\mathrm{HCl}, 150 \mathrm{mM}$ $\mathrm{NaCl}, 2 \mathrm{mM}$ EDTA, $0.35 \% \mathrm{NP}-40$, 0.5\% sodium deoxycholate) containing Complete Protease Inhibitor Cocktail and PhosSTOP Phosphatase Inhibitor Cocktail Tablets (Roche). Cell lysates or liver homogenates were rotated for 30 minutes at $4^{\circ} \mathrm{C}$ prior to removal of cell or tissue debris by centrifugation $(12,000 \mathrm{~g}$ for $20 \mathrm{~min}$ utes). Proteins were denatured by heating for 5 minutes at $96^{\circ} \mathrm{C}$ in Laemmli buffer, separated by SDS-PAGE, and transferred electrophoretically to PVDF membranes (GE Healthcare). Membranes were blocked for 1 hour at $23^{\circ} \mathrm{C}$ in Tris buffer containing $0.1 \%$ Tween-20, 5\% milk, and 1\% BSA. Primary antibodies were then added to the blocking buffer, incubated overnight at $4^{\circ} \mathrm{C}$, detected using goat anti-mouse (Sigma-Aldrich) or anti-rabbit HRP-conjugated secondary antibodies (Bio-Rad), and visualized by enhanced chemiluminescence (GE Healthcare). Images were captured with a Bio-Rad Chemidoc XRS+ system equipped with a digital camera, and densitometry was performed using the histogram analysis function of Adobe Photoshop. Endogenous Them2 was coimmunoprecipitated with PC-TP using Protein A Dynabeads according to the manufacturer's protocol (Invitrogen). Briefly, the protein lysate from the livers of $\mathrm{Pct}^{+/+}$mice was precleared by incubation with $25 \mu \mathrm{l}$ of rabbit IgG-bound beads for 1 hour at $4^{\circ} \mathrm{C}$. IgG-bound beads were discarded, and the precleared lysate was incubated with $25 \mu$ l of PC-TP antibody-bound beads for 2 hours at $37^{\circ} \mathrm{C}$. The protein lysate from the livers of $\mathrm{Pctp}^{-/-}$mice was used as negative control for background Them 2 coimmunoprecipitation. Beads were washed 3 times with PBS before the elution and denaturation of proteins by heating for 5 minutes at $96^{\circ} \mathrm{C}$ in $2 \times$ Laemmli buffer.

Quantitative PCR and reverse transcription PCR analysis of gene expression. mRNA was extracted from cells or tissue using TRIzol (Invitrogen) according to the manufacturer's instructions. cDNA was synthesized from $2 \mu \mathrm{g}$ total mRNA using SuperScript III First-Strand Synthesis System for reverse transcription PCR (Invitrogen). Gene expression was quantified using LightCycler FastStart DNA Master ${ }^{\text {PLus }}$ SYBR Green I (Roche Applied Sciences) in a LightCycler 480II (Roche Applied Sciences). Mouse L32 ribosome protein and TATAA-box binding protein (Tbp) were used as invariant controls $(18,62)$. Nucleotide sequences of oligonucleotide primers were: mouse Pctp, forward 5'-CCAGAGTATCTCGGCACCTC-3' , reverse 5'-ACGCTTTCACCATGTCCTTC-3'; mouse Pemt, forward 5'-TTGGGGATTCGTGTTTGTGCT-3', reverse 5'-CACGCTGAAGGGAAATGTGG-3' (37); mouse Pcyt1a, forward 5'GATGCACAGAGTTCAGCTAAAGT-3', reverse 5'-TGGCTGCCGTAAACCAACTG-3' (37); mouse Pcyt2, forward 5'-TGTGTTCACGGCAATGACATC-3', reverse 5'-TTCCCGGTACTCAGAGGACAT-3' (37); mouse Ptdss1, forward 5'-GCAGGACTCTGAGCAAGGATG-3', reverse 5'-GGCGAAGTACATGAGGCTGAT-3' (37); mouse Ptdss2, forward 5'-GGATTGCCTTTCAGTTCACGC-3', reverse 5'-AGGTAGAAGGTGTTCAGCTCTG-3' (37); mouse Rpl32, forward 5'-CACCAGTCAGACCGATATGT-3', reverse 5'-TTCTCCGCACCCTGTTG-3'; mouse Tbp, forward 5'-ACCCTTCACCAATGACTCCTATG-3', reverse 5'-TGACTGCAGCAAATCGCTTGG-3' (62); human $I P_{3} R 1$, forward 5'-CGCTAAGCCTGGGGCCAA-3', reverse 5'-GGATTACGGTCCCCAGCAAT-3'; human $I P_{3} R 2$, forward 5'-GCCAAACAAGGGAACCACAC-3', reverse 5'-GTATTTTACAATTTCTCCCAACAG-3'; human $I P_{3} R 3$, forward 5'-GACTAAGCAGGACAAGGAGAA-3', reverse 5'-CTTCACGACATCCCCATGCA-3'; human SERCA2A, forward 5'-CCTCTATGTCGAACCCTTGC-3', reverse 5'-GTATTGCAGGTTCCAGGTAGTT-3'; human SERCA2B, forward 5'-CCTCTATGTCGAACCCTTGC-3', reverse 5'-GCAGGCTGCACACACTCTT-3'; human GAPDH, forward 5'-CCTCCCGCTTCGCTCTCT-3', reverse 5'-GGCGACGCAAAAGAAGATG-3'; mouse Grp78, forward 5'-TCATCGGACGCACTTGGAA-3', reverse 5'-CAACCACCTTGAATGGCAAGA-3' (63); mouse Chop, forward 5'-GTCCCTAGCTTGGCTGACAGA-3', reverse 5'-TGGAGAGCGAGGGCTTTG-3' (63).

Statistical analysis. Data are expressed as mean \pm SEM of independent experiments. Means of experimental groups were compared using a 2-tailed Student's $t$ test. Differences were considered statistically significant for $P$ less than 0.05. Statistical analyses were corrected for multiple comparisons when comparing knockdown of PC-TP and Them 2 versus the scrambled siRNA control by a Bonferroni correction so that differences were considered statistically significant for $P$ less than 0.025 .

Study approval. Protocols for animal use, treatment, and euthanasia were approved by the institutional animal care and use committees of Harvard Medical School and Weill Cornell Medical College.

\section{Author contributions}

BAE contributed to the hypothesis, designed and performed experiments, analyzed data, and wrote the manuscript. KMMS designed and performed mass spectrometry experiments and analyzed data. YL performed experiments. IA designed and performed calcium flux experiments. DEC contributed to the hypothesis, directed the work, designed experiments, and wrote the manuscript. 


\section{Acknowledgments}

This work was supported by NIH grants to DEC (DK048873 and DK056626) and BAE (DK102733). DEC was also supported by American Diabetes Association grant 7-13-MI-04. BAE was also the recipient of an NIH Ruth L. Kirschstein National Research Service Award (DK093195) and an American Association for the Study of Liver Diseases Liver Scholar Award.
Address correspondence to: David E. Cohen, Weill Cornell Medical College, 413 East 69th Street, Belfer Research Building, Room BB-630, New York, New York 10021, USA. Phone: 646.962.4746; E-mail: dcohen@med.cornell.edu.

KMMS's present address is: Department of Biochemistry, Emory School of Medicine, Atlanta, Georgia, USA.
1. Hu FB, van Dam RM, Liu S. Diet and risk of Type II diabetes: the role of types of fat and carbohydrate. Diabetologia. 2001;44(7):805-817.

2. Feskens EJ, Kromhout D. Habitual dietary intake and glucose tolerance in euglycaemic men: the Zutphen Study. Int JEpidemiol. 1990;19(4):953-959.

3. Vessby B, et al. Substituting dietary saturated for monounsaturated fat impairs insulin sensitivity in healthy men and women: the KANWU Study. Diabetologia. 2001;44(3):312-319.

4. Borradaile NM, Han X, Harp JD, Gale SE, Ory DS, Schaffer JE. Disruption of endoplasmic reticulum structure and integrity in lipotoxic cell death. JLipid Res. 2006;47(12):2726-2737.

5. Rong X, et al. LXRs regulate ER stress and inflammation through dynamic modulation of membrane phospholipid composition. Cell Metab. 2013;18(5):685-697.

6. Ozcan U, et al. Chemical chaperones reduce ER stress and restore glucose homeostasis in a mouse model of type 2 diabetes. Science. 2006;313(5790):1137-1140.

7. Pagliassotti MJ. Endoplasmic reticulum stress in nonalcoholic fatty liver disease. Annu Rev Nutr. 2012;32:17-33.

8. Alkhouri N, Dixon LJ, Feldstein AE. Lipotoxicity in nonalcoholic fatty liver disease: not all lipids are created equal. Expert Rev Gastroenterol Hepatol. 2009;3(4):445-451.

9. Kawano Y, Cohen DE. Mechanisms of hepatic triglyceride accumulation in non-alcoholic fatty liver disease. J Gastroenterol. 2013;48(4):434-441.

10. Coleman RA, Lewin TM, Van Horn CG, GonzalezBaró MR. Do long-chain acyl-CoA synthetases regulate fatty acid entry into synthetic versus degradative pathways? J Nutr. 2002;132(8):2123-2126.

11. Grevengoed TJ, Klett EL, Coleman RA. Acyl-CoA metabolism and partitioning. Annu Rev Nutr. 2014;34:1-30.

12. Birkenfeld AL, Shulman GI. Nonalcoholic fatty liver disease, hepatic insulin resistance, and type 2 diabetes. Hepatology. 2014;59(2):713-723.

13. Kanno K, et al. Interacting proteins dictate function of the minimal START domain phosphatidylcholine transfer protein/StarD2.J Biol Chem. 2007;282(42):30728-30736.

14. Wei J, Kang HW, Cohen DE. Thioesterase superfamily member 2 (Them2)/acyl-CoA thioesterase 13 (Acot13): a homotetrameric hotdog fold thioesterase with selectivity for long-chain fatty acyl-CoAs. Biochem J. 2009;421(2):311-322.

15. Kang HW, Niepel MW, Han S, Kawano Y, Cohen DE. Thioesterase superfamily member $2 /$ acylCoA thioesterase 13 (Them2/Acot13) regulates hepatic lipid and glucose metabolism. FASEB J. 2012;26(5):2209-2221.

16. Ersoy BA, et al. Phosphatidylcholine transfer protein interacts with thioesterase superfamily member 2 to attenuate insulin signaling. Sci Signal. 2013;6(286):ra64.

17. Shishova EY, et al. Genetic ablation or chemical inhibition of phosphatidylcholine transfer protein attenuates diet-induced hepatic glucose production. Hepatology. 2011;54(2):664-674.

18. Scapa EF, et al. Regulation of energy substrate utilization and hepatic insulin sensitivity by phosphatidylcholine transfer protein/StarD2. FASEB J. 2008;22(7):2579-2590.

19. Kawano Y, Ersoy BA, Li Y, Nishiumi S, Yoshida M, Cohen DE. Thioesterase superfamily member 2 (Them2) and phosphatidylcholine transfer protein (PC-TP) interact to promote fatty acid oxidation and control glucose utilization. Mol Cell Biol. 2014;34(13):2396-2408.

20. Tillander V, Alexson SEH, Cohen DE. Deactivating fatty acids: acyl-CoA thioesterase-mediated control of lipid metabolism. Trends Endocrinol Metab. 2017;28(7):473-484.

21. Ozcan U, et al. Endoplasmic reticulum stress links obesity, insulin action, and type 2 diabetes. Science. 2004;306(5695):457-461.

22. Park SW, et al. The regulatory subunits of PI3K, p $85 \alpha$, and p $85 \beta$, interact with XBP- 1 and increase its nuclear translocation. Nat Med. 2010;16(4):429-437.

23. Wagle N, et al. Small-molecule inhibitors of phosphatidylcholine transfer protein/StarD2 identified by high-throughput screening. Anal Biochem 2008;383(1):85-92.

24. Kirkby B, Roman N, Kobe B, Kellie S, Forwood $J K$. Functional and structural properties of mammalian acyl-coenzyme A thioesterases. Prog Lipid Res. 2010;49(4):366-377.

25. Li WW, Alexandre S, Cao X, Lee AS. Transactivation of the grp78 promoter by $\mathrm{Ca}^{2+}$ depletion. A comparative analysis with $\mathrm{A} 23187$ and the endoplasmic reticulum $\mathrm{Ca}(2+)$-ATPase inhibitor thapsigargin. J Biol Chem. 1993;268(16):12003-12009.

26. Flourakis M, Van Coppenolle F, Lehen'kyi V, Beck B, Skryma R, Prevarskaya N. Passive calcium leak via translocon is a first step for iPLA2-pathway regulated store operated channels activation. FASEB J. 2006;20(8):1215-1217.

27. Hammadi M, et al. Modulation of ER stress and apoptosis by endoplasmic reticulum calcium leak via translocon during unfolded protein response: involvement of GRP78. FASEB J. 2013;27(4):1600-1609.

28. Cassel R, et al. Protection of human pancreatic islets from lipotoxicity by modulation of the translocon. PLoS One. 2016;11(2):e0148686.

29. $\mathrm{Tu} \mathrm{H}$, et al. Presenilins form $\mathrm{ER} \mathrm{Ca}^{2+}$ leak channels, a function disrupted by familial Alzheimer's disease-linked mutations. Cell. 2006;126(5):981-993.

30. Li G, et al. Role of ERO1- $\alpha$-mediated stimulation of inositol 1,4,5-triphosphate receptor activity in endoplasmic reticulum stress-induced apoptosis. J Cell Biol. 2009;186(6):783-792.

31. Coughlin SR. How the protease thrombin talks to cells. Proc Natl Acad Sci U S A. 1999;96(20):11023-11027.

32. Wei Y, Wang D, Gentile CL, Pagliassotti MJ. Reduced endoplasmic reticulum luminal calcium links saturated fatty acid-mediated endoplasmic reticulum stress and cell death in liver cells. Mol Cell Biochem. 2009;331(1-2):31-40.

33. Spector AA, Yorek MA. Membrane lipid composition and cellular function. J Lipid Res. 1985;26(9):1015-1035.

34. Castuma CE, Brenner RR. Effect of fatty acid deficiency on microsomal membrane fluidity and cooperativity of the UDP-glucuronyltransferase. Biochim Biophys Acta. 1983;729(1):9-16.

35. Christon R, et al. The effect of dietary essential fatty acid deficiency on the composition and properties of the liver microsomal membrane of rats. J Nutr. 1988;118(11):1311-1318.

36. Yang X, Sheng W, Sun GY, Lee JC. Effects of fatty acid unsaturation numbers on membrane fluidity and $\alpha$-secretase-dependent amyloid precursor protein processing. Neurochem Int . 2011;58(3):321-329.

37. Fu S, et al. Aberrant lipid metabolism disrupts calcium homeostasis causing liver endoplasmic reticulum stress in obesity. Nature. 2011;473(7348):528-531.

38. Serra D, Mera P, Malandrino MI, Mir JF, Herrero L. Mitochondrial fatty acid oxidation in obesity. Antioxid Redox Signal. 2013;19(3):269-284.

39. El-Said KS, Ali EM, Kanehira K, Taniguchi A. Molecular mechanism of DNA damage induced by titanium dioxide nanoparticles in toll-like receptor 3 or 4 expressing human hepatocarcinoma cell lines. J Nanobiotechnology. 2014;12:48.

40. Ozcan L, et al. Calcium signaling through CaMKII regulates hepatic glucose production in fasting and obesity. Cell Metab. 2012;15(5):739-751.

41. Ozcan L, Cristina de Souza J, Harari AA, Backs J, Olson EN, Tabas I. Activation of calcium/ calmodulin-dependent protein kinase II in obesity mediates suppression of hepatic insulin signaling. Cell Metab. 2013;18(6):803-815.

42. Schuck S, Prinz WA, Thorn KS, Voss C, Walter P. Membrane expansion alleviates endoplasmic reticulum stress independently of the unfolded protein response. JCell Biol. 2009;187(4):525-536.

43. Sriburi R, Jackowski S, Mori K, Brewer JW. XBP1: a link between the unfolded protein response, lipid biosynthesis, and biogenesis of the endoplasmic reticulum. J Cell Biol. 2004;167(1):35-41.

44. Hamman BD, Chen JC, Johnson EE, Johnson AE. The aqueous pore through the translocon has a diameter of 40-60 Å during cotranslational protein translocation at the ER membrane. Cell. 
1997;89(4):535-544.

45. Pierobon N, Renard-Rooney DC, Gaspers LD, Thomas AP. Ryanodine receptors in liver. J Biol Chem. 2006;281(45):34086-34095.

46. Száraz P, Bánhegyi G, Marcolongo P, Benedetti A. Transient knockdown of presenilin-1 provokes endoplasmic reticulum stress related formation of autophagosomes in HepG2 cells. Arch Biochem Biophys. 2013;538(2):57-63.

47. Li Y, et al. Enrichment of endoplasmic reticulum with cholesterol inhibits sarcoplasmic-endoplasmic reticulum calcium ATPase- $2 b$ activity in parallel with increased order of membrane lipids: implications for depletion of endoplasmic reticulum calcium stores and apoptosis in cholesterol-loaded macrophages. J Biol Chem. 2004;279(35):37030-37039.

48. Gibellini F, Smith TK. The Kennedy pathwayde novo synthesis of phosphatidylethanolamine and phosphatidylcholine. IUBMB Life. 2010;62(6):414-428.

49. Bartlett PJ, Gaspers LD, Pierobon N, Thomas AP. Calcium-dependent regulation of glucose homeostasis in the liver. Cell Calcium. 2014;55(6):306-316.

50. Haze K, Yoshida H, Yanagi H, Yura T, Mori K. Mammalian transcription factor ATF6 is synthe- sized as a transmembrane protein and activated by proteolysis in response to endoplasmic reticulum stress. Mol Biol Cell. 1999;10(11):3787-3799.

51. Wu MK, Hyogo H, Yadav SK, Novikoff PM, Cohen DE. Impaired response of biliary lipid secretion to a lithogenic diet in phosphatidylcholine transfer protein-deficient mice. J Lipid Res. 2005;46(3):422-431.

52. Dibble CC, Asara JM, Manning BD. Characterization of Rictor phosphorylation sites reveals direct regulation of mTOR complex 2 by S6K1. Mol Cell Biol. 2009;29(21):5657-5670.

53. Dietrich A, et al. Pressure-induced and storeoperated cation influx in vascular smooth muscle cells is independent of TRPC1. Pflugers Arch. 2007;455(3):465-477.

54. Lytton J, Westlin M, Hanley MR. Thapsigargin inhibits the sarcoplasmic or endoplasmic reticulum Ca-ATPase family of calcium pumps. J Biol Chem. 1991;266(26):17067-17071.

55. Galvagnion C, et al. Lipid vesicles trigger $\alpha$-synuclein aggregation by stimulating primary nucleation. Nat Chem Biol. 2015;11(3):229-234.

56. Cox B, Emili A. Tissue subcellular fractionation and protein extraction for use in massspectrometry-based proteomics. Nat Protoc. 2006;1(4):1872-1878.
57. Bligh EG, Dyer WJ. A rapid method of total lipid extraction and purification. Can J Biochem Physiol.1959;37(8):911-917.

58. Pulfer M, Murphy RC. Electrospray mass spectrometry of phospholipids. Mass Spectrom Rev. 2003;22(5):332-364.

59. Ejsing CS, et al. Automated identification and quantification of glycerophospholipid molecular species by multiple precursor ion scanning. Anal Chem. 2006;78(17):6202-6214.

60. Nicholls HT, Hornick JL, Cohen DE. Phosphatidylcholine transfer protein/StarD2 promotes microvesicular steatosis and liver injury in murine experimental steatohepatitis. Am J Physiol Gastrointest Liver Physiol. 2017;313(1):G50-G61.

61. Wang DD, et al. Plasma ceramides, mediterranean diet, and incident cardiovascular disease in the PREDIMED Trial (Prevención con Dieta Mediterránea). Circulation. 2017;135(21):2028-2040.

62. Choi JH, et al. Antidiabetic actions of a non-agonist PPAR $\gamma$ ligand blocking Cdk5-mediated phosphorylation. Nature. 2011;477(7365):477-481.

63. Zhang Y, et al. Targeted deletion of thioesterase superfamily member 1 promotes energy expenditure and protects against obesity and insulin resistance. Proc Natl Acad Sci U S A. 2012;109(14):5417-5422. 